\title{
Evolution and Regulation of a Large Effector Family of Pyricularia oryzae
}

\author{
Daniel J. Ebbole, ${ }^{1,+}$ Meilian Chen, ${ }^{1,2}$ Zhenhui Zhong, ${ }^{3}$ Nicholas Farmer, ${ }^{1}$ Wenhui Zheng, ${ }^{3}$ Yijuan Han, ${ }^{2}$ \\ Guodong Lu, ${ }^{3}$ and Zonghua Wang ${ }^{2,3,4}$ \\ ${ }^{1}$ Department of Plant Pathology \& Microbiology, Texas A\&M University, College Station, TX, 77843, U.S.A. \\ ${ }^{2}$ Institute of Oceanography, Minjiang University, Fuzhou, 350108, China \\ ${ }^{3}$ State Key Laboratory of Ecological Pest Control for Fujian and Taiwan Crops, College of Plant Protection, Fujian Agriculture \\ and Forestry University, Fujian 350002, China \\ ${ }^{4}$ Fujian Universities Key Laboratory of Plant-Microbe Interactions, College of Life Science, Fujian Agriculture and Forestry \\ University, Fujian 350002, China
}

Accepted 30 October 2020.

Plant pathogen effectors play important roles in parasitism, including countering plant immunity. However, investigations of the emergence and diversification of fungal effectors across host-adapted populations has been limited. We previously identified a gene encoding a suppressor of plant cell death in Pyricularia oryzae (syn. Magnaporthe oryzae). Here, we report the gene is one of a 21-member gene family and we characterize sequence diversity in different populations. Within the rice pathogen population, nucleotide diversity is low, however; the majority of gene family members display presence-absence polymorphism or other null alleles. Gene family allelic diversity is greater between host-adapted populations and, thus, we named them host-adapted genes (HAGs). Multiple copies of $H A G s$ were found in some genome assemblies and sequence divergence between the alleles in two cases suggested they were the result of repeat-induced point mutagenesis. Transfer of family members between populations and novel $H A G$ haplotypes resulting from apparent recombination were observed. $H A G$ family transcripts were induced in planta and a subset of $H A G$ s are dependent on a key regulator of pathogenesis, PMK1. We also found differential intron splicing for some $H A G$ s that would prevent ex planta protein expression. For some genes, spliced transcript was expressed in antiphase with an overlapping antisense transcript. Characterization of $H A G$ expression patterns and allelic diversity reveal novel mechanisms for $H A G$

Current address for Zhenhui Zhong: Department of Molecular, Cell and Developmental Biology, University of California, Los Angeles, CA 90095, U.S.A.

${ }^{\dagger}$ Corresponding author: D. J. Ebbole; d-ebbole@tamu.edu

Funding: This work was funded by the National Natural Science Foundation of China grants U1805232, 31601596, 31770156, and 32001976, the United States Department of Agriculture Hatch Project grant 113944, National Science Foundation grant 0605017, China Scholarship Council, and the National Key R\&D Program of China grant 2016YFD0300700.

*The $\boldsymbol{e}$-Xtra logo stands for "electronic extra" and indicates supplementary materials are published online.

The author(s) declare no conflict of interest.

(c) (1) () $\odot$ Copyright $\odot 2021$ The Author(s). This is an open access article distributed under the CC BY-NC-ND 4.0 International license. regulation and mechanisms generating sequence diversity and novel allele combinations. This evidence of strong in plantaspecific expression and selection operating on the $H A G$ family is suggestive of a role in parasitism.

Keywords: blast disease, gene duplication, horizontal gene transfer (HGT), MGG_17227, parasexual, RIP mutagenesis

Plant pathogens produce effector proteins that can interact with multiple host proteins, and key host proteins may be recognized by many different pathogen effectors (Weßling et al. 2014). Overall, the cumulative action of many individual effectors contributes to lesion number and lesion size in Pyricularia oryzae (Liao et al. 2016). Effector proteins can be recognized by plant resistance proteins to activate plant immunity and such effectors are then also called avirulence (Avr) proteins. Pathogen effectors often have substantial redundancy, and disruption of effector/Avr protein-resistance protein interaction (e.g., by $A V R$ gene loss or silencing) restores virulence, often without obvious loss of aggressiveness. This can result in a host-adapted pathogen population with presence-absence or other null allele polymorphism for effector/AVR genes.

$P$. oryzae parasitizes a wide range of cereal and grass hosts with genetically distinguishable populations adapted to different hosts (Gladieux et al. 2018a and b). Although most isolates belong to these distinct lineages, population analyses using wholegenome sequencing (WGS) have shown that gene flow between populations occurs (Gladieux et al. 2018b). A number of WGS analyses have been carried out to assess mechanisms of evolution driving pathogen diversity and to identify candidate effector genes based on patterns of evolution that suggest effector/AVR gene coevolution with host resistance genes (Chiapello et al. 2015; Kim et al. 2019; Latorre et al. 2020; Petit-Houdenot et al. 2020; Yoshida et al. 2016). Host shifts of P. oryzae to wheat (Inoue et al. 2017) and from rice to Panicum repens (Torpedograss) (Couch and Kohn 2002) have been documented. In the case of the shift to wheat, after the initial invasion of wheat lines lacking resistance, gene loss in the pathogen allowed adaptation to the presence of resistance genes present in other cultivars.

$A V R 1-\mathrm{CO} 39$ is a gene identified as conferring Avr to rice carrying the $\mathrm{Pi}$-CO39 resistance gene. $A V R 1-C O 39$ is common among grass pathogens but functional alleles are absent from rice pathogens. The $\mathrm{Pi}$ - $\mathrm{CO} 39$ resistance gene is not found in all 
rice cultivars, but it has been suggested that it may have been predominant in the location of rice domestication where $P$. oryzae first adapted to rice, thereby selecting for lineages lacking the gene (Tosa et al. 2005).

$P$. oryzae Eleusine and rice pathogen populations contain $P W L 1$ and PWL2, respectively, and, when transferred to weeping lovegrass isolates, these genes cause a loss of pathogenicity to weeping lovegrass (Kang et al. 1995; Sweigard et al. 1995). In nature, one Eleusine pathogen lineage contains $P W L 1$ and this differentiates the two major Eleusine lineages by differentiating host range (Asuke et al. 2020b). At least five $A V R$ genes recognized by wheat were identified in Eleusine isolates (Asuke et al. 2020a). Considering these examples, it is clear that host-adapted pathogen populations may harbor effector genes that function as $A V R$ genes on a different host and lack functional effector/AVR genes recognized by resistance genes in its own host population.

We previously sequenced a population of rice isolates (Zhong et al. 2018), and, in that analysis, we identified a $P$. oryzae gene (gene ID MGG_17227) that displayed presence-absence polymorphism. We further showed the gene is expressed during colonization and delivered into the host cell. Transient coexpression of MGG_17227 and Bax1 protein in tobacco suppressed Bax1-mediated cell death, as has been found for other effectors (Park et al. 2012). As MGG_17227 is one member of a gene family, we hypothesize that all members of the gene family play a role in the interaction with the plant host.

Sequence divergence of effector genes may allow adaptation to different hosts by optimizing interactions with target proteins in the new host (Dong et al. 2014). We do not know if gene family members are involved in host adaptation, but we named the gene family host-adapted genes (HAGs) to describe that different host-adapted populations tended to have different combinations of alleles for the genes. In this study, we examined allelic diversity of the gene family in $P$. oryzae and found evidence for generation of new alleles and found $H A G$ movement between host-adapted populations. The patterns of gene distribution are similar to those described above for cultivar-specific and host species-specific $A V R$ genes.

Effectors are often preferentially expressed during host colonization (Jeon et al. 2020; Torres et al. 2016). We found differential expression of the $H A G$ family in planta and differential regulation by a regulator of pathogenesis, $P M K 1$. In addition, we identified stage-specific intron splicing and detected expression of overlapping antisense RNA for at least three family members. These findings suggest multiple modes of transcriptional and posttranscriptional regulation of $H A G$ family expression.

\section{RESULTS}

The $H A G$ effector gene family in $P$. oryzae.

The $H A G$ family encodes approximately 100 - to 120 -amino acid proteins with predicted secretion signal peptides of 16 to 24 amino acids. The proteins share motifs that are unique to the gene family (Fig. 1), which were used to identify the family members by alignment (Altschul et al. 1990; Brown et al. 1998; Madeira et al. 2019). The order of the motifs is conserved with a cysteinerich region containing four or five cysteine residues, a GCD tripeptide containing the sixth conserved cysteine, a GRxV motif, and a C-terminal $\mathrm{F}(\mathrm{I} / \mathrm{V}) \mathrm{GC}(\mathrm{A} / \mathrm{V})$ motif containing the eighth conserved cysteine. Not all family members have eight cysteines, excluding the predicted signal peptide (Almagro Armenteros et al. 2019), the proteins contain six or eight cysteine residues with the exception of $H A G 17$, which lacks the fifth conserved cysteine residue and so has seven cysteines. Proteins with six cysteine residues lack the fourth and seventh cysteine residues relative to the proteins with eight cysteines (Fig. 1).

All 21 members of the $P$. oryzae $H A G$ family are present in rice isolate P131 (Xue et al. 2012) (Table 1). The genes are dispersed in the genome ( $>20 \mathrm{~Kb}$ apart), with the exception of $H A G 19$ and $H A G 20$, which are separated by less than $2 \mathrm{~Kb}$. A comparison of $H A G$ locations in the reference $P$. oryzae strain 70-15 (Dean et al. 2005), Eleusine isolate MZ5-1-6 (Gómez Luciano et al. 2019), and Triticum isolate B71 (Peng et al. 2019) showed a good degree of synteny (Fig. 2). It was shown that the $\mathrm{S} 8$ segment of the $70-15$ reference genome corresponds to one end of chromosome 7 in $\mathrm{B} 71$, indicating that $H A G 18$ is syntenic in these isolate genomes (Peng et al. 2019). Supplementary Table S1 provides the locations, orientation, and distances between the genes. A translocation of a $1.9-\mathrm{Mb}$ segment of chromosome 6 to chromosome 1 in $70-15$ was noted previously (Gómez Luciano et al. 2019; Peng et al. 2019) and this

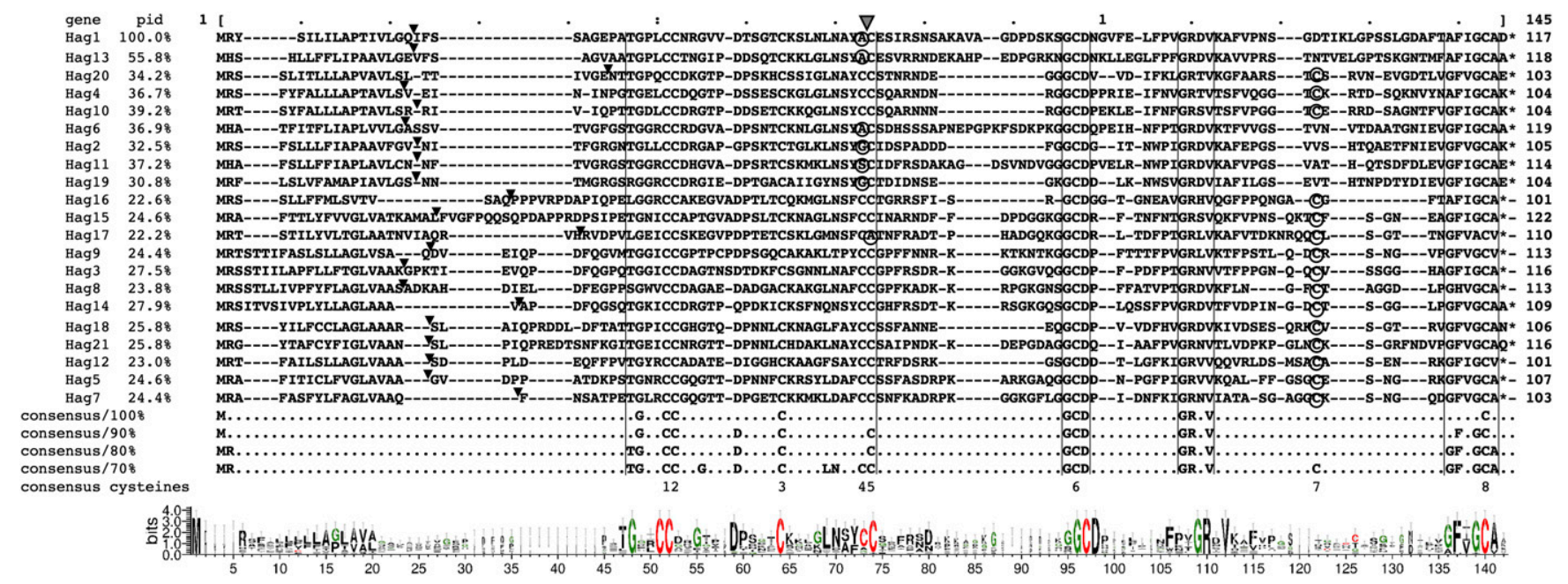

Fig. 1. Alignment of Pyricularia oryzae host-adapted gene (HAG) proteins. Sequence identity to Hag1p is shown (pid). Smaller arrowheads point to the predicted signal peptide cleavage sites. Indicated regions highlight a cysteine-rich, GCD tripeptide, GRxV, and C-terminal FI/VGCA motifs. Consensus sequences with 100 to $70 \%$ identity are indicated in the lines below the alignment with conserved cysteines numbered below. Circled residues highlight three types of proteins. The six-cysteine group has substitutions at position 4 (circled) and lack the cysteine at position 7 relative to those with eight cysteines (position 7 circled). Hag17p lacks cysteine 5 (circled alanine substitution) and has just seven cysteines. An intron in the genomic sequences lies precisely between the codons specifying cysteines 4 and 5 in all genes (large arrowhead above Hag 1 p sequence). A WebLogo graphic representation is at bottom. 
accounts for HAG6 residing on chromosome 1 in $70-15$ and chromosome 6 in MZ5-1-6 and B71. We noted copies of HAG4 ( $H A G 4 b$ and $H A G 4 c$ ) on chromosomes 1 and 6 are roughly near the new and old location of HAG6 in 70-15 (Fig. 2). The absence of some family members in 70-15 (HAG19 to HAG21), MZ5-1-6 (HAG4, HAG21), and B71 (HAG4), and the multiple copies of $H A G 4$ in 70-15 did not alter the conclusion that $H A G$ organization in the genome is largely maintained. The $H A G 4 c$ allele differs from HAG4a and HAG4b by one SNP (single nucleotide polymorphism) and has the same sequence as the reference allele in P131. Supplementary Table S2 provides the DNA and protein sequences used as reference sequences and Supplementary Table S3 is a compendium listing the genome assemblies assessed and the SNP differences from the reference alleles for each gene for each genome.

There is considerable sequence variation between paralogs, for example, alignments of Hag1p with other family members were found to have sequence identity ranging from 22 to $56 \%$ (Fig. 1), and, in overall pairwise comparisons, the greatest identity is $73 \%$ between Hag4p and Hag10p.

Of the 18 family members present in the 70-15 genome, automated annotation of the 70-15 reference assembly correctly predicted nine of them, three were not predicted as coding regions, and six gene models were incorrect. Thus, previous transcriptome studies generally would not have been able to fully assess expression of all members of the family. In addition to manual annotation of the two exons and intron for each gene, the assemblies for the genes that had rare SNPs or indels were assessed, if possible. For example, the sequence assemblies for $H A G 3$ required correction in two important genome assemblies. The HAG3 sequence in the 70-15 reference genome assembly GCF_000002495.2 has an erroneous extra cytosine (C) residue yielding a stretch of eight $\mathrm{C}$ residues that predicts a frameshift in the coding sequence. Examination of the

Table 1. Gene family location in the P131 (SAMN02981399; AHZT01) assembly

\begin{tabular}{|c|c|c|c|c|c|}
\hline Broad ID ${ }^{\mathbf{a}}$ & Gene name & Contig & Orientation $^{\mathbf{b}}$ & Start codon & Stop codon \\
\hline MGG_17227 & $H A G 1$ & AHZT01002070.1 & $\mathrm{F}$ & 14,867 & 15,292 \\
\hline MGG_17240 & $H A G 2^{\mathrm{c}}$ & AHZT01002549.1 & $\mathrm{R}$ & 1,573 & 1,178 \\
\hline MGG_16603 & HAG3 & AHZT01000287.1 & $\mathrm{R}$ & 1,701 & 1,262 \\
\hline MGG_17587 & HAG4 & AHZT01000776.1 & $\mathrm{R}$ & 852 & 472 \\
\hline MGG_16814 & $H A G 5^{\mathrm{c}}$ & AHZT01000450.1 & $\mathrm{R}$ & 13,393 & 12,974 \\
\hline MGG_16404 & $H A G 6^{\mathrm{c}}$ & AHZT01001918.1 & $\mathrm{F}$ & 770 & 1,208 \\
\hline MGG_17155 & HAG7 & AHZT01000892.1 & $\mathrm{R}$ & 1,095 & 1,516 \\
\hline MGG_10732 & $H A G 8$ & AHZT01002379.1 & $\mathrm{R}$ & 41,170 & 40,740 \\
\hline MGG_16401 & HAG9 & AHZT01001367.1 & $\mathrm{F}$ & 2,637 & 3,078 \\
\hline MGG_05403 & HAG10 & AHZT01001637.1 & $\mathrm{R}$ & 8,198 & 7,803 \\
\hline MGG_07352 & HAG11 & AHZT01000147.1 & $\mathrm{F}$ & 19,573 & 20,022 \\
\hline MGG_15924 & HAG12 & AHZT01001332.1 & $\mathrm{F}$ & 7,505 & 7,914 \\
\hline MGG_16703 & HAG13 & AHZT01002050.1 & $\mathrm{R}$ & 4,322 & 3,894 \\
\hline None & $H A G 14$ & AHZT01001360.1 & $\mathrm{R}$ & 15,882 & 15,449 \\
\hline None & HAG15 & AHZT01001973.1 & $\mathrm{R}$ & 2,696 & 2,265 \\
\hline MGG_17205 & HAG16 & AHZT01000006.1 & $\mathrm{R}$ & 1,189 & 800 \\
\hline None & HAG17 & AHZT01001450.1 & $\mathrm{F}$ & 1,417 & 1,819 \\
\hline MGG_18102 & HAG18 & AHZT01002202.1 & $\mathrm{F}$ & 12,801 & 13,238 \\
\hline None & HAG19 & AHZT01000264.1 & $\mathrm{R}$ & 1,310 & 928 \\
\hline None & HAG2O & AHZT01000264.1 & $\mathrm{R}$ & 3,241 & 2,856 \\
\hline None & $H A G 21$ & AHZT01000088.1 & $\mathrm{F}$ & 2,929 & 3,362 \\
\hline
\end{tabular}

${ }^{a}$ Broad ID from 70-15 assembly GCF_000002495.2.

${ }^{\mathrm{b}} \mathrm{F}$ and $\mathrm{R}$ indicate forward or reverse gene orientation.

${ }^{\mathrm{c}}$ Contains frameshift mutations in all rice isolates.
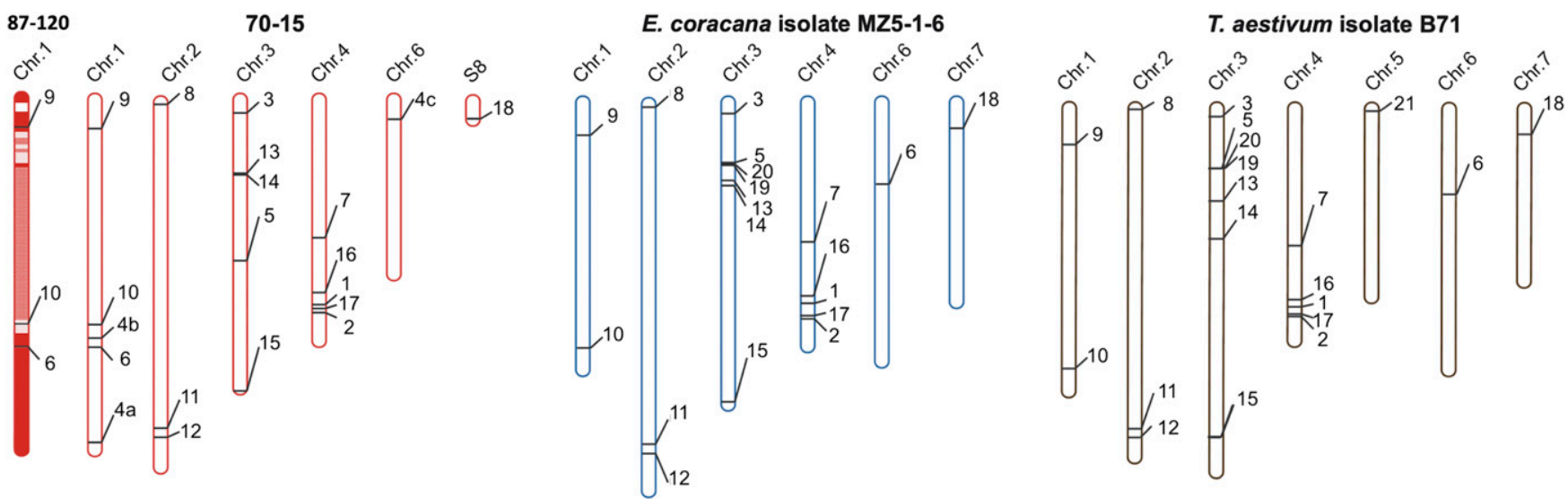

Fig. 2. Schematic of host-adapted gene $(H A G)$ distribution in the reference genome assemblies and lineage assessment of chromosome 1 of isolate $87-120$. Rice isolate 70-15 lacks HAG19 and HAG20, which are located near HAG5 in MZ5-1-6 and B71. Isolates 70-15 and MZ5-1-6 both lack HAG21, which is located near the tip of chromosome 5 in B71. Isolate 70-15 has three copies of HAG4 (designated HAG4a, HAG4b, and HAG4c). MZ5-1-6 and B71 lack HAG4. Rice isolate 87-120 was shown to be a recombinant with DNA aligning best to sequences of different lineages (Gladieux et al. 2018a and b). Chromosome 1 aligns with segments of single-copy sequence with near identity to 70-15 (solid color), G17 (light shading), MZ5-1-6 (medium shading), or different from these three sequences (no shading near tip). HAG10 lies within a $263-\mathrm{Kb}$ region of near identity with G17 sequence. 
deposited sequence chromatograms and fastq data unambiguously shows seven Cs at that position in all deposited reads, yielding the correct reading frame (discussed below). The B71 genome assembly GCA_004785825.1 (200× genome coverage) misplaced approximately $120 \mathrm{bp}$ covering the $5^{\prime}$ end of HAG3 (present in the raw reads, absent in the assembly). However, the smaller GCA_001675625.1 assembly of B71 (157× genome coverage) was correct for the HAG3 region. We manually annotated each predicted rare SNP or indel if sequencing reads were deposited with the assemblies.

\section{Rice isolate allelic diversity.}

The major rice pathogen lineage is proposed to have diverged from a $P$. oryzae Setaria-adapted population about 10,000 years ago (approximately 1,200 to 22,000) (Couch and Kohn 2002; Gladieux et al. 2018a), and the current population is estimated to have had a common origin as recently as about 1,000 years ago (175 to 2,700) (Gladieux et al. 2018a; Latorre et al. 2020; Zhong et al. 2018). Not surprisingly, there is generally low sequence diversity within the $P$. oryzae rice-adapted population, although there is clear evidence for genetic exchange between populations (Gladieux et al. 2018b).

Frameshift alleles for $H A G 2, H A G 5$, and HAG6 are fixed in the rice pathogen population, except for a small number of isolate sequences from which the genes are absent. HAG2 has a deletion of a CTTT sequence at nucleotide 19 in the protein coding sequence, HAG5 has an insertion of a CCCT sequence after nucleotide 78, and HAG6 has a deletion of a CA dinucleotide at position 308. The Setaria viridis isolate SV9610 genome sequence does not have frameshifted alleles but otherwise is identical to rice isolate alleles for $H A G 2$ and HAG5 and has one synonymous SNP for HAG6. The SV9610 isolate sequences for these genes were used for protein sequence comparison with other family members (Fig. 1; Supplementary Table S2) and were used as the reference sequence for SNP analysis. We confined our SNP analysis to the coding regions to simplify the analysis, and intron variation was not considered in defining alleles.

Rice isolate 87-120 was previously shown to be a recombinant (Gladieux et al. 2018b) and this isolate is discussed later. Of the remaining 150 rice isolate genome sequences across all 21 genes, only 13 different SNPs were identified (Supplementary Table S3). Disregarding four SNPs in the HAG2, $H A G 5$, and HAG6 pseudogenes, altered protein products would result from four nonsynonymous substitutions: N53D $(n=1)$ in $H A G 7, \mathrm{~A} 47 \mathrm{~V}(n=3)$ in HAG12, and S3F $(n=2)$ and F8I $(n=$ $35)$ in HAG13.

A WGS SNP-based tree was used to place 90 rice isolates into three clades (Fig. 3) (Zhong et al. 2018). Clade 3 represents a clonal lineage of Mat1-1 isolates and Clade 2 represents a clonal lineage of Mat1-2 isolates. Clade 1 was assigned to capture the remaining genetic diversity of the rice pathogens and contains isolates of both mating types. Just ten of these 90 genome sequences contain all 21 members of the gene family, and these are all in clade 3 . Thirty-one of the 37 remaining clade 3 isolates belong to a lineage lacking HAG15. HAG1 and $H A G 21$ are absent in all 28 clade 2 isolate sequences. The 15 clade 1 isolates each lack from one to six family members. Overall, 13 family members (HAG1 to HAG5, HAG9, HAG12, $H A G 15, H A G 16, H A G 18$ to $H A G 21)$ display presence-absence polymorphism among these 90 assemblies. The phylogenetic pattern of gene presence and absence indicates that deletions of some genes (e.g., HAG21) have resulted from independent events (Fig. 3).

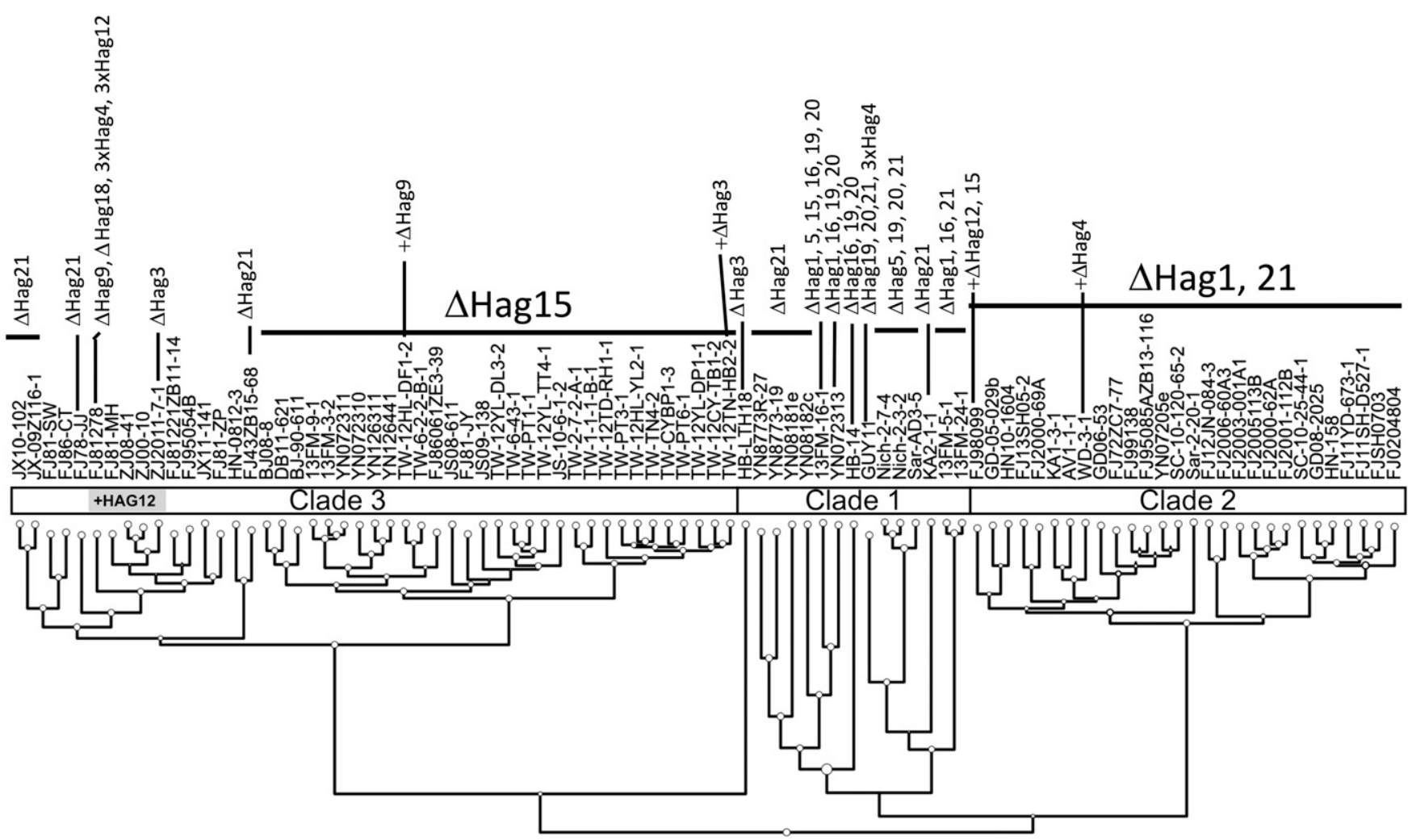

Fig. 3. Relationship between host-adapted gene $(H A G)$ family deletions and rice isolate phylogeny. Whole-genome sequencing (WGS) single nucleotide polymorphism tree with isolates assigned to three clades. Gene deletions are indicated (horizontal bars indicate multiple isolates containing the same deletion). Other deletions are labeled over vertical lines. Genes deleted in addition to those indicated by horizontal bars are indicated with a plus (+) sign. Genes occurring in triplicate copies are indicated (3x). Isolates related to FJ81278 in clade 3 containing more than one copy of HAG12 are indicated with a gray box ( $H A G 12$ ). 
Five genes (HAG7, HAG8, HAG10, HAG13, and HAG17) were present in all 151 rice isolate genome sequences examined, HAG18 was absent in one sequence, and HAG4, HAG6, $H A G 12$, and HAG14 were each absent from two genome sequences (Supplementary Table S3). In FJ81278, the HAG9 and HAG18 deletions (Fig. 3) are each captured within single contigs and the genes were replaced by transposon insertions. Relative to Guy11 as a reference, an approximately 1,800-bp Fot 1 repetitive element (Kito et al. 2003) replaced a 23,418-bp region containing $H A G 9$. A single approximately 1,860-bp copy of a Pot2 transposon (Kimura and Yamaguchi 1998) replaced a 8,345-bp region containing HAG18. Contigs often terminate at repetitive element sequences so that deletion endpoints are unclear in most cases.

The median number of $H A G$ deletions per rice isolate is 1 (average 1.7, standard deviation 1.3). The greatest number of deleted HAGs in an isolate is six for 13-FM-16-1. We noted that the B157 and RMg-DI genome assemblies lacked seven and 12 $H A G$ s, respectively, however, we excluded those assemblies from this analysis (discussed below).

In isolates from other host populations, frameshifts, repetitive element insertions, and gene deletions are also observed, yielding

Table 2. Analysis of the KJ201 infection time course and Guy11 PMK1-dependent $H A G$ expression data $^{\mathrm{a}}$

\begin{tabular}{|c|c|c|c|c|c|c|c|c|c|c|c|c|}
\hline \multirow[b]{3}{*}{ Gene } & \multicolumn{6}{|c|}{ Data from Jeon et al. (2020) } & \multicolumn{6}{|c|}{ Reanalysis } \\
\hline & \multicolumn{6}{|c|}{ Hours postinoculation } & \multicolumn{6}{|c|}{ Hours postinoculation } \\
\hline & Mycelia & 18 & 27 & 36 & 45 & 72 & Mycelia & 18 & 27 & 36 & 45 & 72 \\
\hline \multicolumn{13}{|c|}{ FPKM values of the KJ201 infection time course } \\
\hline$H A G 1$ & 0.0 & 2.5 & 6.5 & 7.1 & 28.5 & 5.9 & 0.1 & 2.9 & 16.0 & 6.7 & 29.7 & 7.2 \\
\hline$H A G 2$ & 0.0 & 42.0 & 67.6 & 54.9 & 59.3 & 9.2 & 0.0 & 42.6 & 177 & 57.7 & 62.3 & 12.2 \\
\hline HAG3 & 0.0 & 43.2 & 54.1 & 103 & 244 & 121 & 0.0 & 58.4 & 163 & 123 & 307 & 186 \\
\hline HAG4 & 0.0 & 9.4 & 38.5 & 4.3 & 4.2 & 3.2 & 0.0 & 43.9 & 140 & 16.6 & 14.7 & 14.0 \\
\hline HAG5 & 0.1 & 14.2 & 6.1 & 14.7 & 10.5 & 6.4 & 0.0 & 13.5 & 23.7 & 14.5 & 15.9 & 11.3 \\
\hline HAG6 & 0.0 & 0.0 & 0.3 & 0.0 & 0.4 & 0.8 & 0.1 & 0.5 & 0.6 & 0.4 & 0.5 & 1.3 \\
\hline$H A G 7$ & 0.2 & 20.9 & 16.4 & 24.4 & 13.1 & 6.3 & 0.7 & 21.8 & 43.0 & 19.7 & 11.4 & 1.9 \\
\hline$H A G 8$ & 0.0 & 2.3 & 1.1 & 1.7 & 3.4 & 1.0 & 0.0 & 2.3 & 4.6 & 2.0 & 3.5 & 1.4 \\
\hline$H A G^{9}$ & 0.0 & 0.0 & 0.0 & 0.0 & 0.0 & 0.0 & 0.0 & 8.6 & 79.6 & 27.0 & 2.9 & 0.3 \\
\hline HAG10 & 0.0 & 9.4 & 20.2 & 6.1 & 11.6 & 2.6 & 0.0 & 12.9 & 38.0 & 10.6 & 16.5 & 4.5 \\
\hline HAG11 & 0.0 & 0.3 & 0.0 & 0.0 & 0.0 & 0.0 & 0.0 & 0.3 & 0.0 & 0.2 & 0.0 & 0.0 \\
\hline HAG12 & 0.0 & 11.1 & 31.9 & 85.8 & 247 & 64.4 & 0.0 & 9.6 & 72.0 & 65.4 & 196 & 61.8 \\
\hline HAG13 & 0.0 & 44.8 & 88.2 & 5.9 & 8.8 & 5.1 & 0.5 & 39.9 & 130 & 5.4 & 8.5 & 7.5 \\
\hline HAG14 & N/A & N/A & N/A & N/A & N/A & N/A & 0.0 & 7.4 & 22.1 & 3.0 & 2.5 & 2.3 \\
\hline HAG15 & \multicolumn{6}{|c|}{ Gene absent } & \multicolumn{6}{|c|}{ Gene absent } \\
\hline HAG16 & 0.1 & 15.4 & 12.0 & 23.9 & 16.9 & 17.5 & 0.1 & 16.1 & 36.1 & 23.3 & 16.1 & 22.2 \\
\hline HAG17 & N/A & N/A & N/A & N/A & N/A & N/A & 0.0 & 20.2 & 50.2 & 6.4 & 3.7 & 2.6 \\
\hline HAG18 & 0.0 & 0.0 & 0.0 & 0.0 & 9.7 & 10.6 & 0.0 & 14.7 & 25.4 & 17.3 & 41.0 & 56.5 \\
\hline HAG19 & N/A & N/A & N/A & N/A & N/A & N/A & 0.0 & 286.8 & 369 & 156 & 368 & 155 \\
\hline HAG2O & N/A & N/A & N/A & N/A & N/A & N/A & 0.0 & 113.1 & 359 & 123 & 164 & 54.9 \\
\hline AvrPi9 & 3.4 & 855 & 1,930 & 1,560 & 1,650 & 462 & 9.3 & 1,050 & 3,066 & 1,806 & 2,191 & 833 \\
\hline \multirow[t]{2}{*}{ AvrPiz-t } & 0.0 & 18.2 & 36.5 & 41.2 & 63.6 & 51.6 & 0.2 & 29.9 & 129 & 60.4 & 116 & 115 \\
\hline & \multicolumn{6}{|c|}{ Data from Sakulkoo et al. (2018) } & \multicolumn{6}{|c|}{ Reanalysis } \\
\hline Gene & Control & & 1NA-PP1 & $Q$ value & & sulated ${ }^{\mathbf{b}}$ & Control & & PP1 & Regulated $^{\mathrm{b}}$ & & \\
\hline \multicolumn{13}{|c|}{ FPKM values for chemical inhibition of $P M K 126$ to $32 \mathrm{~h}$ postinfection } \\
\hline HAG1 & 9.05 & & 8.04 & 1 & & No & 15.7 & & .5 & No & & \\
\hline$H A G 2$ & 0.00 & & 0.00 & 1 & & No & 3.3 & & .0 & No & & \\
\hline HAG3 & 11.12 & & 0.00 & 1 & & No & 180 & & .3 & Yes & & \\
\hline HAG4 & 0.00 & & 0.00 & 1 & & No & 130 & & 8 & No & & \\
\hline HAG5 & N/A & & N/A & N/A & & N/A & 105 & & .0 & Yes & & \\
\hline HAG6 & 0.00 & & 0.00 & 1 & & No & 0.0 & & .0 & No & & \\
\hline HAG7 & 0.00 & & 2.08 & 1 & & No & 2.1 & & .2 & No & & \\
\hline HAG8 & 10.09 & & 0.00 & 1 & & No & 9.3 & & .0 & No & & \\
\hline$H A G 9$ & N/A & & N/A & N/A & & N/A & 2.0 & & .0 & No & & \\
\hline HAG10 & 11.59 & & 0.00 & 1 & & No & 9.8 & & .0 & No & & \\
\hline HAG11 & 0.00 & & 0.00 & 1 & & No & 0.0 & & .0 & No & & \\
\hline$H A G 12$ & 128.24 & & 0.00 & 0.0005 & & Yes & 93.9 & & .2 & Yes & & \\
\hline HAG13 & 9.88 & & 4.84 & 1 & & No & 11.4 & & 7.6 & No & & \\
\hline HAG14 & N/A & & N/A & N/A & & N/A & 2.1 & & .0 & No & & \\
\hline HAG15 & N/A & & N/A & N/A & & N/A & 0.5 & & .6 & No & & \\
\hline HAG16 & 22.37 & & 0.00 & 0.001 & & Yes & 9.9 & & .3 & No & & \\
\hline$H A G 17$ & N/A & & N/A & N/A & & N/A & 5.4 & & 1.3 & No & & \\
\hline HAG18 & 0.00 & & 0.00 & 1 & & No & 14.4 & & .7 & No & & \\
\hline HAG19 & \multicolumn{6}{|c|}{ Gene absent } & \multicolumn{6}{|c|}{ Gene absent } \\
\hline HAG2O & \multicolumn{6}{|c|}{ Gene absent } & \multicolumn{6}{|c|}{ Gene absent } \\
\hline AvrPi9 & 72.83 & & 0.00 & 0.444 & & No & 439 & & 2.6 & No & & \\
\hline AvrPiz-t & 0.00 & & 3.09 & 1 & & No & 0 & & .2 & No & & \\
\hline
\end{tabular}

${ }^{a}$ FPKM $=$ fragments per kilobase per million reads. N/A = not analyzed, not reported, or no value.

b PMK1 was judged as regulated with a minimum of 30 hits to the gene across experiments and $>10$-fold difference. 
additional null alleles for Hag1, Hag4 to Hag7, Hag9, Hag12, Hag13 to Hag 15, Hag17 to Hag 18, and Hag20 (Supplementary Table S3). Overall, of the 21 HAGs, null alleles for only HAG8 and $H A G 10$ have not yet been identified in natural isolates.

Genome assemblies with multiple copies of HAGs were found. As with 70-15, three copies of HAG4 were found in the FJ81278 and Guy11 genome sequences (Bao et al. 2017) (Fig. 3). Two copies of HAG4 were also present in the V86010 assembly and all other rice isolate assemblies examined had one copy. Because the Guy11 and FJ81278 assemblies were based on long read (PacBio) sequences (Bao et al. 2017), the detection of multiple copies of HAG4 might be due to the enhanced ability to assemble multiple gene copies surrounded by large regions of repetitive sequence. Therefore, we reevaluated the raw sequencing reads for 95 genome assemblies and found a distribution of 11 isolates with the reference allele (P131) sequence only, 52 isolates with the t213c allele only, and 32 isolates with sequencing reads showing both alleles present. All three genotypes were found distributed across clade 3 , the $\mathrm{t} 213 \mathrm{c}$ allele alone or both allele types were present in clade 1 isolates, and all clade 2 isolates uniformly contained only the $\mathrm{t} 213 \mathrm{c}$ allele. Southern blotting was used to verify multiple copies in Guy11, 70-15, and FJ81278, and single copies in FJ98099, Sar-2-20-1, and FJ72ZC7-77 (data not shown). Three copies of HAG12 were also found in FJ81278 in clade 3 (Fig. 3), with one copy having no SNPs relative to P131 and two copies having a c140t SNP. We examined sequencing reads from isolates most closely related to FJ81278 (FJ78-JJ to FJ81-ZP) (Fig. 3). We found both alleles present in FJ81-MH, ZJ08-41, ZJ00-10, and ZJ2011-7-1.

Deletion and duplication events may be aided by flanking repetitive elements that promote recombination (Yoshida et al. 2016), and genes for secreted effector proteins appear to be concentrated in regions with above-average density of transposon sequences in P. oryzae (Zhong et al. 2020). The majority of $H A G$ family members in the sequences of FJ81278 and Guy11 have repetitive elements within $5 \mathrm{~kb}$ either upstream, downstream, or upstream and downstream of the gene. The three copies of HAG4 in rice isolates have closely flanking repetitive elements (range around 450 to around 1,500 bp). The three copies of HAG12 in FJ81278 also have relatively short distances to repetitive elements (around 1.4 to around $8.9 \mathrm{~Kb}$ ).

The closeness of some repetitive sequences to the coding region may impact gene expression. For $H A G 17$, repetitive sequences lie approximately $270,6,600$, and $31,000 \mathrm{bp}$ upstream of the start codon in FJ81278, KJ201, and Guy11, respectively. In the case of the HAG6 pseudogene in the sequence of KJ201 and other isolates, a repetitive sequence lies just 395 bp upstream of the start codon.

\section{Expression and differential regulation of $\boldsymbol{H A G}$ family members.}

As some HAGs were not previously annotated, we reassessed a published transcriptome time course of infection with rice isolate KJ201 (Jeon et al. 2020). HAG15 and HAG21 are absent from the KJ201 genome sequence and no transcripts were found for them (Table 2). Data for HAG14, HAG17, HAG19, and $H A G 20$ were not reported in the previous study, as there were no gene predictions for them. In addition, differences in our gene models for HAG3, HAG5, HAG6, HAG7, HAG16, and $H A G 18$ and our inclusion of 150-bp flanking regions affected expression values in some cases. In both analyses, HAG6 and HAG11 were poorly expressed.

The peak of expression for most gene family members was found at 27 or $45 \mathrm{~h}$ postinfection (hpi), during the main stage of biotrophic growth. This timing was consistent with expression peaks for AvrPi9 and AvrPiz-t (Table 2). Transcript levels declined more rapidly from the 27 hpi peak for some genes
( $>5$-fold decrease in expression at 36 hpi for HAG4, HAG13, $H A G 14$, and HAG17). HAG1, HAG2, HAG5, HAG7 to HAG10, HAG16, HAG19, and HAG20 showed a slower decline in expression, HAG3 and HAG12 peaked at 45 hpi and $H A G 18$ was peaking at $72 \mathrm{hpi}$. Thus, family members do not share a single common expression pattern during host colonization, suggesting they may play coordinated but distinct roles during host colonization.

To further assess regulation of the gene family, we reexamined published transcriptome data (Sakulkoo et al. 2018) to determine the requirement for $P M K 1$ mitogen-activated protein kinase. $P M K 1$ regulates pathogenicity and it was found that a number of effector genes were dependent on $P M K 1$ activity. The study used an engineered allele of $P M K 1$ sensitive to chemical inhibition by 1NA-PP1 and compared gene expression after $6 \mathrm{~h}$ of treatment with the inhibitor after an initial infection period of $26 \mathrm{~h}$. Guy11 was the parental strain and $H A G 19$ to $H A G 21$ are absent from the genome. Expression of eight $H A G$ genes, HAG1, HAG3, HAG7, HAG8, HAG10, HAG12, HAG13, and HAG16 was detected previously (Sakulkoo et al. 2018). We detected expression of $16 H A G$ family members (Table 2). HAG12 and HAG16 were determined to be regulated by $P M K 1$ in the original study (Table 2). In our reanalysis, we required a minimum of 30 mRNA fragments per gene and 10-fold differential expression as a signal for regulation by $P M K 1$ and found $H A G 3$, the $H A G 5$ pseudogene, and $H A G 12$ to be regulated by PMK1. HAG8, HAG10, HAG16, and $H A G 18$ may also be dependent on $P M K 1$; however, the number of detected transcripts for these genes was low. HAG4, HAG13, $H A G 15$, and $H A G 17$ were not regulated by $P M K 1$, thus, $H A G$ family members are regulated differentially by $P M K 1$.

\section{Unspliced and antisense $H A G$ transcript abundance.}

All members of the gene family contain a single intron precisely between the codons specifying the fourth and fifth consensus cysteine residues. Analysis of reads from the KJ201 infection time course that align across the junction of the intron demonstrated the presence of unspliced transcript. We also noted alternative splicing for several HAGs, but these were of low abundance $(<1 \%)$. Overall, the lowest levels of spliced transcripts were found for $H A G 1$ with $84 \%$ (34 unspliced, 173 spliced), HAG13 with $92 \%$ (34 unspliced, 402 spliced), and HAG17 with $63 \%$ (39 unspliced, 65 spliced) combined across all timepoints. At $72 \mathrm{hpi}$, these values were $88 \%$ (65 of 74 spliced), $76 \%$ (60 of 79), and 0\% (0 of 20). AvrPi9 has an intron and this transcript displayed $99 \%$ spliced introns across the time course and $99 \%$ spliced at $72 \mathrm{hpi}$. Sixteen transcripts for AvrPi9 were found in the in-vitro mycelial sample and we noted seven unspliced and nine spliced transcripts. There were very few $H A G$ family fragments crossing the intron boundary in the mycelial samples, but for $H A G 1, H A G 7, H A G 13$, and $H A G 16$, there were a combined 12 reads and all were unspliced. For the first intron in the actin transcript, we found $>98 \%$ spliced transcripts in the infection time course and $>99 \%$ in mycelia.

In addition to unspliced RNA, we noted a relatively abundant antisense transcript with an 84-nucleotide (nt) intron overlapping HAG17. The antisense transcript was expressed in antiphase with spliced $H A G 17$ transcripts. HAG17 displayed efficient splicing at 18 and $27 \mathrm{hpi}$, with 65 spliced, five unspliced, and three spliced antisense RNA fragments combined. In contrast, no spliced HAG17 transcripts were detected at 36,45 , or 72 hpi, but 34 unspliced and 14 antisense spliced transcripts were detected. The unspliced transcripts could be either from the sense or antisense transcripts. We also noted two reads with a 97-bp antisense transcript intron for $H A G 13$ at 72 hpi and one antisense read at 72 hpi for HAG1. As with $H A G 17$, we found relatively few unspliced transcripts for 
HAG13 until 45 hpi (31 spliced, 11 unspliced) and 72 hpi (44 spliced, 28 unspliced, two antisense). The HAG1, HAG13, and $H A G 17$ antisense transcript introns are similarly positioned and overlap the $H A G$ transcript introns.

We assessed two transcriptome studies of in vitro-grown cells with sufficient sequencing depth to detect intron splicing for $12 H A G$ s. One study evaluated the role of protein kinase $\mathrm{C}$ (PKC) on gene expression in mycelia of Guy11 (Penn et al. 2015). The second study examined the role of a transcription factor, $V R F 1$, on gene expression $5 \mathrm{~h}$ after induction of appressoria in the 70-15 background (Cao et al. 2016). Intron splicing was efficient in the first intron of actin, with nearly $100 \%$ of transcripts spliced (Table 3). Including genes with at least 20 RNA fragment sequences aligning to the intron region, the combined data indicate overall splicing of $H A G 2, H A G 4$, HAG5, HAG6, and HAG9 was efficient (70 to $100 \%$ spliced). HAG16 and AvrPi9 were spliced with moderate efficiency (40 to $60 \%$ ). Transcripts were spliced at low frequency (0 to $15 \%)$ for HAG3, HAG7, HAG10, HAG13, HAG15, and HAG17. Thus, there is differential splicing of $H A G$ family member transcripts or differential production of unspliced antisense transcript, when grown ex planta.

For $H A G 17$, in addition to the 373 unspliced fragments (Table 3), an additional 89 spliced antisense transcript fragments were detected, representing apprpoximately $20 \%$ of the total transcripts. This ratio is similar to that observed in the 36to 72-hpi timepoints in the KJ201 infection study. Analysis of the potential HAG17 region antisense transcript based on the

Table 3. Splicing of host-adapted gene $(H A G)$ introns of in-vitro mycelia and appressoria

\begin{tabular}{|c|c|c|c|c|c|c|c|}
\hline \multirow[b]{2}{*}{ Gene } & \multicolumn{2}{|c|}{ PKC study } & \multicolumn{2}{|c|}{ Vrf1 Study } & \multicolumn{3}{|c|}{ Combined } \\
\hline & Splice & No Splice & Splice & No Splice & Splice & No Splice & $\%$ Spliced \\
\hline$H A G 1$ & 0 & 1 & 0 & 0 & 0 & 1 & N/A \\
\hline HAG2 & 0 & 1 & 27 & 0 & 27 & 1 & 96 \\
\hline HAG3 & 4 & 26 & 2 & 137 & 6 & 163 & 4 \\
\hline HAG4 & 108 & 4 & 1,878 & 26 & 1,986 & 30 & 99 \\
\hline HAG5 & 115 & 29 & 461 & 1 & 576 & 30 & 95 \\
\hline HAG6 & 16 & 5 & 0 & 2 & 16 & 7 & 70 \\
\hline$H A G 7$ & 15 & 307 & 0 & 6 & 15 & 313 & 5 \\
\hline HAG8 & 0 & 2 & 1 & 4 & 1 & 6 & 14 \\
\hline$H A G 9$ & 1 & 1 & 15 & 4 & 16 & 5 & 76 \\
\hline HAG1O & 0 & 3 & 68 & 462 & 68 & 465 & 13 \\
\hline HAG11 & 0 & 0 & 0 & 0 & 0 & 0 & N/A \\
\hline HAG12 & 0 & 0 & 0 & 0 & 0 & 0 & N/A \\
\hline HAG13 & 0 & 44 & 0 & 0 & 0 & 44 & 0 \\
\hline HAG14 & 0 & 2 & 1 & 3 & 1 & 5 & 17 \\
\hline HAG15 & 4 & 20 & 2 & 182 & 6 & 202 & 3 \\
\hline HAG16 & 31 & 22 & 1 & 23 & 33 & 45 & 42 \\
\hline HAG17 & 0 & 4 & 2 & 369 & 2 & 373 & 0.5 \\
\hline HAG18 & 0 & 0 & 2 & 7 & 2 & 7 & 22 \\
\hline AvrPi9 & 206 & 150 & 10 & 10 & 216 & 160 & 57 \\
\hline Actin & 2485 & 6 & 20,909 & 18 & 23,394 & 24 & 100 \\
\hline
\end{tabular}

Table 4. Allele number, allele SNPs or indels and predicted protein changes for $H A G 11$ and $H A G 13^{\mathrm{a}}$

\begin{tabular}{|c|c|c|c|c|}
\hline \multirow[b]{2}{*}{ Allele } & \multicolumn{2}{|l|}{ HAG11 } & \multicolumn{2}{|c|}{ HAG13 } \\
\hline & SNP alleles $=16$ & Hag11p variants $=11$ & SNP alleles $=15$ & Hag13p variants $=11$ \\
\hline 0 & Full deletion & N/A & No deletion allele & N/A \\
\hline 1 & None & No change & None & No change \\
\hline 2 & $\mathrm{~g} 135 \mathrm{a}$ & No change & $\mathrm{c} 8 \mathrm{t}$ & $\mathrm{S} 3 \mathrm{~F}$ \\
\hline 3 & $\mathrm{t} 279 \mathrm{c}$ & No change & $\mathrm{c} 24 \mathrm{~g}$ & F8L \\
\hline 4 & $\mathrm{c} 60 \mathrm{t}$ & No change & $\mathrm{c} 8 \mathrm{t}$ a51g & F8L \\
\hline 5 & $\mathrm{c} 60 \mathrm{t} \mathrm{c} 261 \mathrm{~g}$ & No change & $\mathrm{c} 8 \mathrm{t} \mathrm{a} 28 \mathrm{~g}$ & S3F I10V \\
\hline 6 & $\mathrm{c} 110 \mathrm{a}$ & A37E & c8t a28g Del 212a & S3F I10V frameshift \\
\hline 7 & c110a a154g t161c t165e g170a & A37E S52G I54T R57K & $\mathrm{c} 8 \mathrm{t}$ a28g t $291 \mathrm{c}$ & S3F I10V \\
\hline 8 & $\begin{array}{l}\mathrm{c} 110 \mathrm{a} \text { a154g t161 t } 166 \mathrm{c} \text { c177t } \\
\text { c237g c246t }\end{array}$ & A37E S52G I54T F56I & $\begin{array}{l}\text { c8t c83a a103g a119c c191g } \\
\text { t250g g271t g320t }\end{array}$ & $\begin{array}{l}\text { S3F P28Q I35V Q40P P64R F84V } \\
\text { V91F G107V }\end{array}$ \\
\hline 9 & t107c c110a a154g t161c g170a a289g & $\begin{array}{l}\text { V36A A37E S52G I54T } \\
\text { R57K T97A }\end{array}$ & $\begin{array}{l}\text { c8t c83a a103g c191g t250g } \\
\text { g271t }\end{array}$ & S3F P28Q I35V P64R F84V V91F \\
\hline A & $\begin{array}{l}\mathrm{t} 107 \mathrm{c} c 110 \mathrm{a} \text { a154g t161c t } 213 \mathrm{c} \\
\mathrm{c} 246 \mathrm{t}\end{array}$ & V36A A37E S52G I54T & $\begin{array}{l}\text { c8t c83a a103g c191g t250g } \\
\text { g271t g320t }\end{array}$ & $\begin{array}{l}\text { S3F P28Q I35V P64R F84V V91F } \\
\text { G107V }\end{array}$ \\
\hline B & $\begin{array}{l}\text { a5g c54t c110a t127g a154g t161c } \\
\text { g170a }\end{array}$ & $\begin{array}{l}\text { H2R A37E C43G S52G } \\
\text { I54T R57K }\end{array}$ & $\begin{array}{l}\text { c8t t30c t36g a51c g52a c66g } \\
\text { t68a c83a a131c c190a c191g } \\
\text { t250g g271t c333t c353a }\end{array}$ & $\begin{array}{l}\text { S3F E17D V18I V23E P28Q K44T } \\
\text { P64Q F84V V91F }\end{array}$ \\
\hline $\mathrm{C}$ & $\begin{array}{l}\text { c54t c110a t127g a154g t161c } \\
\text { g170a }\end{array}$ & $\begin{array}{l}\text { A37E C43G S52G I54T } \\
\text { R57K }\end{array}$ & $\begin{array}{l}\text { c8t t30c t36g a51c g52a t68a c83a } \\
\text { a131c c190a c191g t250g g271t } \\
\text { c333t c353a }\end{array}$ & $\begin{array}{l}\text { S3F E17D V18I V23E P28Q K44T } \\
\text { P64Q F84V V91F }\end{array}$ \\
\hline $\mathrm{D}$ & $\begin{array}{l}\mathrm{c} 54 \mathrm{t} \mathrm{t} 107 \mathrm{c} \text { c110a a154g t161c t165c } \\
\mathrm{g} 170 \mathrm{a}\end{array}$ & $\begin{array}{l}\text { V36A A37E S52G I54T } \\
\text { R57K }\end{array}$ & c8t a51g c83a c150g g271t a292g & SF3 E17D P28Q Y50* V91F T98A \\
\hline E & $\begin{array}{l}\text { c54t c110a t127g a154g t161c g170a } \\
\quad \text { a181g }\end{array}$ & $\begin{array}{l}\text { A37E C43G S52G I54T } \\
\text { R57K K61E }\end{array}$ & c8t a51g c83a g271t a292g & SF3 E17D P28Q V91F T98A \\
\hline $\mathrm{F}$ & $\begin{array}{l}\text { t44c c110a a154g t161c t165c g170a } \\
\text { c261g }\end{array}$ & $\begin{array}{l}\text { V15A A37E S52G I54T } \\
\text { R57K }\end{array}$ & $\mathrm{c} 8 \mathrm{t} \mathrm{c} 16 \mathrm{t}$ & SF3 L6F \\
\hline
\end{tabular}

\footnotetext{
${ }^{a}$ Bold indicates conserved Cys or nonsense single nucleotide polymorphism (SNP).
} 
Table 5. Alleles ${ }^{\mathrm{a}}$ assigned for $H A G 1$ to $H A G 21$ associated with host and number of isolates in each group

\begin{tabular}{|c|c|c|c|c|c|c|c|c|c|c|c|}
\hline Host & $H A G 1$ & $H A G 2$ & $H A G 3$ & HAG4 & HAG5 & HAG6 & HAG7 & $H A G 8$ & HAG9 & HAG10 & HAG11 \\
\hline Oryza sativa (rice) & 0.1 & 0.2 & 0.1.2.8 & 0.1 .2 & 0.2 .3 .4 .5 & 0.2 & 1.2 & 1 & 0.1 .7 & 1.6 & 0.1.2.3 \\
\hline Setaria viridis & 3.4 & 1.4 & 1 & 3 & 1 & 1 & 3 & 1 & 1 & 1 & 4.6 \\
\hline Setaria italica & 1.4.C & 1 & 1.4 & 3.4 .5 .6 & 1.B & 1 & 3 & 1 & 1 & 1.8 & 6 \\
\hline Panicum repens & 1 & 1 & 1 & 2 & $\mathbf{C}$ & 3 & 3 & 5 & 8 & 1 & 5 \\
\hline Brachiaria distachya (Bd8401) & 8 & 1 & 1 & $\mathbf{0}$ & 1 & 4 & 4 & 5 & 1 & 1 & 1 \\
\hline Eleusine coracana & 7 & 6 & 5 & $\mathbf{0}$ & 7.9.D & 7 & 1 & 2 & 5 & 5 & 7.A \\
\hline Eleusine indica & 7 & 6 & 5 & $\mathbf{0}$ & 7.9.D & 7.8 & 1.8 & 2.4 & 3.5 & 5 & 7.A \\
\hline Eragrostis curvula & $\mathrm{A}$ & 7 & 5 & $\mathbf{0}$ & 6 & 8 & 9 & 3 & 3 & 6 & 9.A \\
\hline Lolium perenne & 6 & 6 & 3 & $\mathbf{0}$ & 7 & 7 & 1 & 2 & 2 & 5 & 1 \\
\hline Lolium multiflorum & 6 & 6 & 3.5 & $\mathbf{0}$ & 7 & 7 & 1 & 2 & 2 & 5 & 1 \\
\hline Lolium sp. & 6 & 6 & 3 & $\mathbf{0}$ & 7 & 7 & 1 & 2 & 2 & 5 & 1 \\
\hline Festuca arundinacea & 6 & 6 & 5 & $\mathbf{0}$ & 7 & 7 & 1 & 2 & 2 & 5 & 1 \\
\hline Triticum aestivum ${ }^{\mathrm{b}}$ & 2.6 & 3.6 & 3.5 & $\mathbf{0}$ & 7.A & 7 & 1.6 & 1.2 & 2 & 3 & 1.D*. $\mathrm{F}^{*}$ \\
\hline Bromus tectorum (P28) & 2 & 3 & 5 & $\mathbf{0}$ & 7 & 7 & 1 & 1 & 2 & 5 & 1 \\
\hline Brachiaria mutica (Bm88324) & 5 & 5 & 4 & $\mathbf{0}$ & 6 & 5 & 7 & 2 & 4 & 4 & 8 \\
\hline Stenotaphrum secundatum & 2 & 3 & 6 & $\mathbf{0}$ & 7 & 6 & 4 & 2 & 4 & 3 & B \\
\hline Cenchrus sp. (MG07) & 9 & 8 & 7 & 0 & 7 & A & B & 6 & 6 & 7 & $\mathrm{C}$ \\
\hline Uncertain host (pg1213-2) & $\mathrm{B}$ & $9^{*}$ & 1 & $\mathbf{0}$ & 7 & 9 & A & 3 & 9 & 2 & $\mathrm{E}$ \\
\hline \multicolumn{12}{|c|}{ (Continued on next page) } \\
\hline
\end{tabular}

${ }^{a}$ Alleles (designated 0 through $\mathrm{F}$ ) are separated by periods; bold = null alleles; italics $=$ alleles unique to these groupings; asterisks $\left({ }^{*}\right)$ indicate nonsynonymous substitution of conserved cysteine residue; NA = incomplete sequence.

${ }^{\mathrm{b}}$ Contains isolates of both Lolium and Triticum genetic lineages and the P29 isolate originally isolated from Bromus spp.

RNA contig, assuming the 84-nt intron separates two exons, suggested the potential to encode a 98-amino acid hypothetical protein that did not possess a predicted signal peptide (Supplementary Fig. S1). Reads defining the locations of the antisense introns for $H A G 1, H A G 13$, and $H A G 17$ are listed in Supplementary Table S4.

\section{Allelic diversity of the $H A G$ family in host-adapted populations.}

Different $P$. oryzae host-adapted populations form genetically distinct lineages, although cross-infection by some isolates is observed. Allelic diversity of HAGs across populations was assessed. Genes with the least allelic diversity affecting the protein sequences across all the isolates were HAG19 (no nonsynonymous SNPs) and HAG8 and HAG14, in which several alleles are found across populations but protein-coding regions were altered by no more than one amino acid. The largest number of alleles were found for HAG11 (16 alleles) and HAG13 (15 alleles), that each yielded 11 protein sequence variants. Allelic diversity across populations (Supplementary Table S3) was compiled to define the set of alleles for each gene (Supplementary Table S5). Supplementary Table S5 entries for HAG11 and HAG13 without designation of host population are shown in Table 4 . The greatest number of amino-acid changes in one of the HAG11 alleles was six (Stenotaphrum sp.) and for HAG13 this was nine (Stenotaphrum and Cenchrus spp.). Some of these alleles may encode nonfunctional proteins as some alleles of HAG11 encode a C43G substitution, which would alter the third conserved cysteine. HAG13 has a frameshift allele, which was verified by manual annotation, and one allele contains a nonsense mutation, Y50*, that is not validated. The greatest number of amino-acid differences were found for alleles of HAG3, HAG6, and HAG9, having 12, 10, and 11 amino-acid changes (approximately 10\% protein sequence divergence).

Table 5 compiles the information of Supplementary Table S5 to summarize alleles present in isolates in each of the hostadapted groups surveyed. The 12 members of the Lolium group (isolates from Lolium species and Festuca arundinacea) were fixed for alleles at 16 of the 20 genes present in the population. The 11 isolates in the Eleusine lineage were fixed for alleles at eight of the 20 genes. Five genes (HAG2, HAG10, HAG13, $H A G 19$, and $H A G 20$ ) were fixed for the same allele across both
Lolium and Eleusine lineages (Table 5). The two populations were fixed for different alleles of HAGl (one amino-acid change) and HAG12 (four amino-acid changes).

The wheat-infecting population contains isolates that belong to Triticum and Lolium genetic lineages. Seven HAGs have fixed alleles in the wheat-infecting population, including the wheat pathogen P29 that was originally isolated from a Bromus host (Pieck et al. 2017). In addition to the null allele for $H A G 14$ found in all wheat-infecting isolates, most wheat isolates ( 25 of 29), have a frameshift mutation for HAG15. Overall, the wheat pathogens are moderately diverse, with the 29 genome sequences analyzed represented by $15 H A G$ haplotypes. Two of the 15 haplotypes are shared by the Lolium multiflorum isolates PL2-1 and PL3-1 (not shown).

Although there are too few isolate sequences for most populations to know if the trend of different host-adapted populations having distinguishable $H A G$ haplotypes will hold, $H A G$ haplotypes may be helpful in assigning isolates to major P. oryzae clades (Fig. 4). For example, the HAG2, HAG5, and HAG6 pseudogenes identify rice-adapted isolates and a Pyret transposon insertion in HAG19 is found exclusively in all Setaria isolate sequences examined thus far. Since gene flow between populations occurs (Gladieux et al. 2018b), exceptions to these patterns are inevitable. However, some $H A G$ alleles may help in assigning isolates to populations.

Multicopy $H A G$ genes arise from gene transfer processes.

Of the two copies of HAG21 in the Festuca isolate TF05-1, one copy is identical to the most common allele in closely related Lolium isolates and most Triticum isolates. The second copy is a unique allele but shares 15 of 16 SNPs with the singlecopy gene found in the Triticum isolate $\mathrm{Br} 7$ and in isolates from other hosts (Table 6). Other isolates do not have this second copy, including the Triticum isolate PY5010, which, based on WGS analysis, is closely related to TF05-1, displaying just 65 SNPs per megabase (Gladieux et al. 2018b) (Fig. 4), does not have this second copy. Chromosome painting of Br7 (Gladieux et al. 2018b) showed that chromosome 5 contains substantial similarity to the Stenotaphrum lineage and the HAG21 region is nearly identical to Stenotaphrum sequences from about $95 \mathrm{~Kb}$ upstream to about $4 \mathrm{~Kb}$ downstream of the gene. The corresponding chromosome painting of TF05-1 does not show any 
Table 5. (Continued from previous page)

\begin{tabular}{|c|c|c|c|c|c|c|c|c|c|c|c|}
\hline Host & HAG12 & HAG13 & HAG14 & $H A G 15$ & HAG16 & HAG17 & HAG18 & HAG19 & HAG20 & $H A G 21$ & Isolates \\
\hline Oryza sativa (rice) & $\mathbf{0 . 1} .2$ & 1.2.3.4 & $\mathbf{0 . 1}$ & $\mathbf{0 . 1 . 2}$ & $\mathbf{0 . 1} .2$ & 1 & 0.1 .8 & $\mathbf{0 . 1}$ & $\mathbf{0 . 1}$ & $\mathbf{0 . 1}$ & 151 \\
\hline Setaria viridis & 0.1 & 2.6 .7 & 1 & 1.2 & 1 & 9 & 0.1 & 1 & 1 & 0.1.4.5 & 4 \\
\hline Setaria italica & 1 & 2.5 & 1 & 0.3 & 1 & 9 & 0.1 & 1 & 1.3 & 4.5 & 3 \\
\hline Panicum repens & 1 & $\mathbf{E}$ & 6 & $\mathbf{0}$ & 1 & 8 & $\mathbf{0}$ & 4 & 2 & 4 & 2 \\
\hline Brachiaria distachya (Bd8401) & 1 & D & 6 & 1 & 1 & 1 & 7 & 4 & 2 & 4 & 1 \\
\hline Eleusine coracana & 4 & 8 & 3.4 .5 & 6.8 & 1.3 .4 & 4.6 & 4.6 & 3 & 1 & 0.8.B & 5 \\
\hline Eleusine indica & 4 & 8 & 3.4 .5 & 6.8 & 1.3 .4 & 4.6 & 4.6 & 3 & 1 & 0.3.8. & 6 \\
\hline Eragrostis curvula & 4 & 9 & 7 & 6 & 5 & 5 & 3 & 3 & 4 & 2 & 1 \\
\hline Lolium perenne & 1 & 8 & 5 & 8 & 4.6 & 6 & 6 & 3 & 1 & 9 & 5 \\
\hline Lolium multiflorum & 1 & 8 & 5 & 6.8 & 4.6 & 6 & 6 & 3 & 1 & 9 & 3 \\
\hline Lolium sp. & 1 & 8 & 5 & 8 & 4.6 & 6 & 6 & 3 & 1 & 9 & 2 \\
\hline Festuca arundinacea & 1 & 8 & 5 & 6.8 & 3.6 & 6 & 6 & 3 & 1 & 9.A & 2 \\
\hline Triticum aestivum $^{\mathrm{b}}$ & 1.3 & 8 & 5 & 4.5 .6 & 3.4 .6 & 3.6 & 2.6 .9 & 3 & 1 & 7.9 & 29 \\
\hline Bromus tectorum (P28) & 1 & 8 & 5 & 5 & 3 & 3 & 6 & 3 & 1 & 9 & 1 \\
\hline Brachiaria mutica (Bm88324) & 5 & NA & 7 & 7 & 6 & 2 & 5.C.D & 4 & 2 & 7 & 1 \\
\hline Stenotaphrum secundatum & 3 & A.B & A & $9^{*}$ & 3 & 3 & 9.C & 3 & 1 & 7 & 2 \\
\hline Cenchrus sp (MG07) & 6 & $\mathrm{C}$ & 8 & A & 7 & 7 & 2 & 5 & 5 & 6 & 1 \\
\hline Uncertain host (pg1213-2) & 7 & NA & B & $\mathrm{B}$ & 6 & $\mathbf{0}$ & A & 3 & 4 & $\mathrm{C}$ & 1 \\
\hline
\end{tabular}

large region of the genome with strong similiarity to the Stenotaphrum lineage; however, a region from about $9 \mathrm{~Kb}$ upstream to about $2 \mathrm{~Kb}$ downstream of HAG 21 copy 2 is nearly identical to the Br7 and Stenotaphrum sequences. This finding supports the view that the second copy is an acquisition by gene transfer and not the result of gene duplication and divergence or whole genome recombination with lineages represented in this study.

Stenotaphrum isolate SSFL14-3 has two complete HAG13 sequences. The copy 1 allele is unique but similar to an allele found in Eragrostis populations and somewhat similar to Eleucine and Lolium populations (Table 6). Copy 2 differs by 14 SNPs from copy 1 but differs by just 1 SNP from the Cenchrus isolate MG07 allele. This suggests that the copies were not generated by duplication and divergence within the Stenotaphrum lineage and that one copy arrived from another lineage.

There are three copies of HAG18 in the Bm88324 assembly, two copies in Stenotaphrum isolate SSFL14-3 and one copy in SSFL02-1 (Table 6; Fig. 5). Copy 1 of SSFL14-3 is identical to the single copy in SSFL02-1 in a 9-Kb region of 5' flanking sequence. Copy 1 of Bm88324 shares $98 \%$ identity $(8,895$ of $9,057 \mathrm{nt})$ across the region. Surprisingly, the Stenotaphrum copy 1 allele is nearly identical (9,054 of 9,057 identical, 99.97\%) to the B71 Triticum isolate sequence and the other Triticum isolates bearing this allele ( 25 of 29 isolates). Thus, in conflict with the WGS phylogeny (Fig. 4), the Stenotaphrum copy 1 and B71 HAG18 gene regions are more closely related to each other than to any other lineages. This region of near identity for $H A G 18$ extends from about $70 \mathrm{~Kb}$ upstream to about $4 \mathrm{~Kb}$ downstream of the gene. Interestingly, chromosome 7 of the Br7 Triticum lineage isolate has substantial identity with Stenotaphrum across the chromosome (Gladieux et al. 2018b). The region of identity runs from about $90 \mathrm{~Kb}$ upstream to about $80 \mathrm{~Kb}$ downstream of the gene. This pattern of mulitiple lengths of the segments aligning with Stenotaphrum lines in the Triticum lineage is consistent with recombination between a Stenotaphrum lineage and a progenitor of the Triticum lineage and subsequent recombination within the lineage.

Rather than the $98 \%$ identity between Bm88324 and Stenotaphrum copy 1 alleles, copy 2 of Bm88324 HAG18 is nearly identical to copy 2 of SSFL14-3 in a $2.5-\mathrm{Kb}$ region containing HAG18 and $5^{\prime}$ flanking sequence (2,546 of 2,548 identical). Copy 1 and copy 2 in SSFL14-3 align only across a 500-bp sequence containing the coding region and share $95.6 \%$ identity (480 of 502 identical). The identity of Stenotaphrum copy 1 with the Triticum allele and copy 2 with copy 2 of Bm88324
(Table 6) make it clear that at least one gene transfer event has occurred. We suggest the direction of transfer was from the Stenotaphrum isolate population to the Triticum isolate population (Fig. 5).

\section{Gene duplication and divergence}

through repeat-induced point mutagenesis (RIP).

We noted that all the SNPs that differentiate the Bm88324 HAG18 copies from each other were $\mathrm{C}$ to $\mathrm{T}$ or $\mathrm{G}$ to A transition mutations (Table 6) and assessed this region for the possibility these alleles were generated by RIP (Ikeda et al. 2002). RIP has been characterized in several fungal species, including

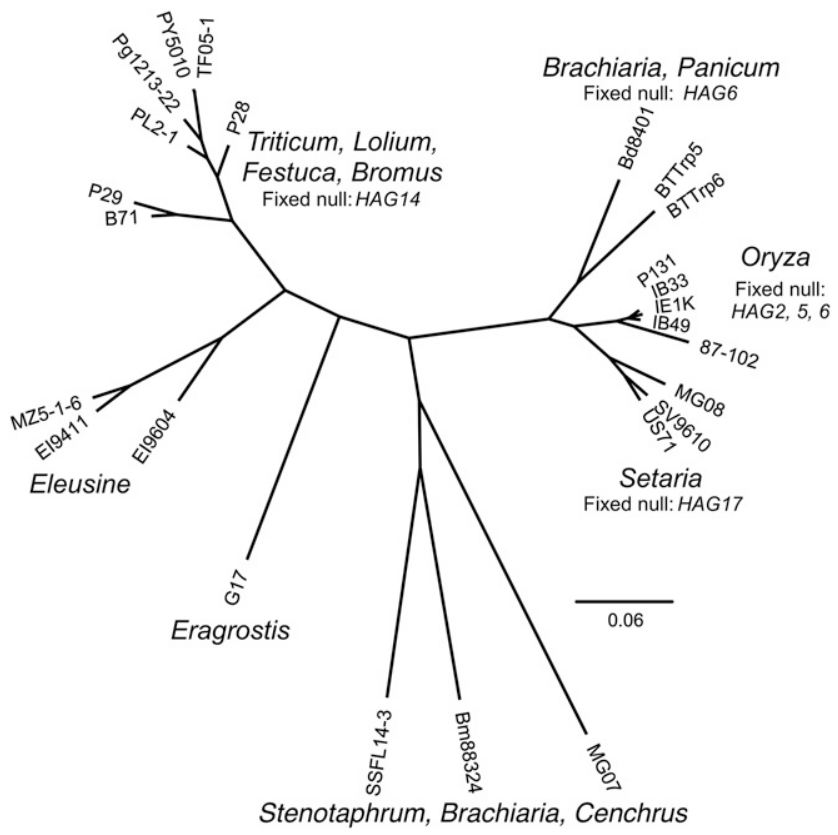

Fig. 4. Whole genome sequencing (WGS) single nucleotide polymorphism (SNP) analysis-based phylogeny of selected members of Pyricularia oryzae populations illustrating diverse lineages. Oryza, Setaria, and Panicum isolates have $H A G 4$, which is absent from genome sequences from all other lineages. Triticum and Lolium isolates are fixed for a null allele in HAG14 that is variably present in the Eleusine lineage. Brachiaria isolate Bd8401, and Panicum repens isolates have SNP variants of the frameshift allele of HAG6 present in the Oryza pathogen population. The Oryza population is also fixed for frameshift alleles of HAG2 and HAG5. The Setaria population sequences are fixed for an insertional of a transposon in HAG17. 
P. oryzae, and this process results in premeiotic CG to TA transition mutations in repeated DNA sequences during mating. We compared Bm88324 HAG18 copies 2 and 3 to each other and they align across a $2.1-\mathrm{Kb}$ region with $95.5 \%$ identity $(2,036$ of 2,100 identities) and we found 61 CG to TA transitions and three other types of SNPs. Although the number of SNPs is low, the ratio of CG to TA transitions is elevated relative to the SNPs between Bm88324 copy 1 and SSFL14-3 copy 1 , which share $98 \%$ identity (93 CG to TA versus 65 other). We propose that copies 2 and 3 are products of RIP mutagenesis. Since the alleles appear to have originated in the Bm88324 lineage, copy 2 must have been transferred to the Stenotaphrum lineage (Fig. 5).

Setaria isolate MG08 has three distinct copies of HAG4 (Table 7). MG05 is a closely related isolate that has three similar copies, but none of these were completely contained in a single contig. The three copies of the gene in MG08 had eight to 11 SNPs in the coding region relative to Setaria isolate US71 and most were GC to TA transitions. To better assess the SNPs in the region, we aligned sequencing reads from the MG05 and MG08 sequencing projects, using the HAG4 region of the US71 genome assembly as a template. An approximate 3,420-bp region bounded by repetitive sequences about 2,650 bp upstream and about 590 bp downstream of the HAG4 start codon in US71 resolved to three copies for each isolate. The coding regions for copies 1 and 2 of each isolate were identical but copy 3 of MG05 differed from copy 3 of MG08 at five positions (Table 7). SNPs relative to US71 that were not fixed across alleles were counted. For MG08, there were $138 \mathrm{CG}$ to TA SNPs versus five other SNPs and, for MG05, this was 130 to five (Table 7). The preponderance of CG to TA transitions in these alleles is indicative of RIP. The alleles have intact coding regions with three to four nonsynonymous
SNPs each, however, one allele has an intron splice site consensus sequence mutation.

\section{Recombinant $H A G$ haplotypes.}

Using chromosome painting analysis (Lawson et al. 2012), it was previously shown that rice isolate $87-120$ is a recombinant mostly similar to the typical rice isolate but having large blocks of DNA with high identity to Eleusine (e.g., MZ5-1-6) and Eragrostis (e.g., G17) genome sequences (Gladieux et al. 2018 b). Twenty of the 21 HAGs in 87-120 were identical to rice isolate alleles. The HAG10 allele is identical to the allele from Eragrostis isolate G17, which has eight SNPs relative to the rice isolate allele. Consistent with the chromosome painting analysis, we found single-copy DNA sequences of 87-120 chromosome 1 aligned to a nearly $5-\mathrm{Mb}$ region that is highly similar (about $100 \%$ identity) to Eleusine isolate MZ5-1-6, and this region is flanked on one side by an approximately $250-\mathrm{Kb}$ region of interleaved Eleusine and Eragrostis lineage segments and on the other side by an approximately $263-\mathrm{Kb}$ segment highly similar (approximately $100 \%$ identity) to the Eragrostis isolate G17 sequence. The HAG10 gene (position $5.106 \mathrm{Mb}$ in 70-15) aligns within this region of high identity to G17 genome sequence (Fig. 2). The recombination junction between Eleusine lineage sequence to Eragrostis lineage sequence near HAG10 occurs in single-copy sequence at about position 5,345,260 in MZ5-1-6 chr 1 (approximately $5.017 \mathrm{Mb}$ in $70-15 \mathrm{chr} 1$, approximately $96.25 \mathrm{~Kb}$ in 87-120 contig PQBK01001703.1). The recombination junction between Eragrostis lineage DNA to Oryza lineage DNA occurs in single-copy sequence at about position $5.2802 \mathrm{Mb}$ in $70-15(14.23 \mathrm{~Kb}$ in $87-120$ contig PQBK01001922.1). Thus, HAG10 is located in the approximately 263$\mathrm{Kb}$ Eragrostis lineage segment.

Table 6. Sequence comparison of multicopy gene alleles for HAG21, HAG13, and HAG18

\begin{tabular}{|c|c|c|}
\hline Gene/host & Isolate (copy \#) & Allele (SNPs relative to P131) ${ }^{a}$ \\
\hline \multicolumn{3}{|l|}{$H A G 21$} \\
\hline $\begin{array}{l}\text { Triticum (28 of } 29 \text { isolates) Festuca } \\
\text { arundinacea }\end{array}$ & PY5010 TF05-1 (copy 1) & $\mathrm{t} 252 \mathrm{c}$ \\
\hline F. arundinacea & TF05-1 (copy 2) & $\begin{array}{l}\text { g8c t19c t21c c33t g54a c55a t57g c58t a61c t144c } \\
\text { c153t c156t g165c } \\
\text { a204g a301c g339a }\end{array}$ \\
\hline $\begin{array}{l}\text { T. aestivum, Brachiaria mutica, } \\
\text { Stenotaphrum secundatum }\end{array}$ & Br7, SSFL14-3, Bm88324 & $\begin{array}{l}\text { g8c t19c t21c c33t g54a c55a t57g c58t a61c t144c } \\
\text { c153t c156t g165c } \\
\text { a204g g339a }\end{array}$ \\
\hline Cenchrus & & $\begin{array}{l}\text { g8c t19c t21c c33t g54a c55a t57g c58t a61c t144c } \\
\text { c153t c156t g165c a204g c251g g 339a }\end{array}$ \\
\hline \multicolumn{3}{|l|}{ HAG13 } \\
\hline Eleucine, Lolium, Triticum & All & $\begin{array}{l}\text { c8t c83a a103g a119c c191g t250g } \\
\text { g271t g320t }\end{array}$ \\
\hline Eragrostis & G17 & $\begin{array}{l}\text { c8t c83a a103g c191g } \\
\text { t250g g271t }\end{array}$ \\
\hline S. secundatum & SSFL14-3 (copy 1) & $\begin{array}{l}\text { c8t c83a a103g c191g } \\
\text { t250g g271t g320t }\end{array}$ \\
\hline S. secundatum & SSFL14-3 (copy 2) & $\begin{array}{l}\text { c8t t30c t36g a51c g52a c66g t68a c83a } \\
\text { a131c c190a c191g t250g g271t c333t c353a }\end{array}$ \\
\hline Cenchrus & MG07 & $\begin{array}{l}\text { c8t t30c t36g a51c g52a t68a c83a } \\
\text { a131c c190a c191g t250g g271t c333t c353a }\end{array}$ \\
\hline \multicolumn{3}{|r|}{ 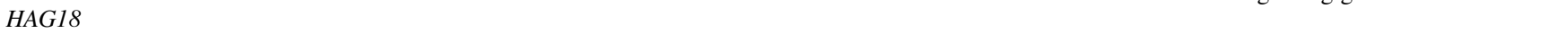 } \\
\hline Triticum (Lolium lineage) & PY5010 & c41t a61g c96g t156c \\
\hline B. mutica, S. secundatum & Bm88324 (copy 2) SSFL14-3 (copy 2) & $\begin{array}{l}\text { c41t g166a g199a g232a g237a } \\
\text { c243t g253a g280a c318t }\end{array}$ \\
\hline B. mutica & Bm88324 (copy 3) & $\begin{array}{l}\text { c41t g196a g232a g237a c243t } \\
\text { g244a g278a g280a c318t }\end{array}$ \\
\hline S. secundatum, $T$. aestivum & $\begin{array}{l}\text { SSFL14-3 (copy 1) SSFL02-1 25/29 } \\
\text { Lolium and Triticum wheat isolates }\end{array}$ & $\mathrm{c} 243 \mathrm{t} \mathrm{c} 255 \mathrm{~g} \mathrm{c} 318 \mathrm{t}$ \\
\hline B. mutica & Bm88324 (copy 1) & $\mathrm{c} 318 \mathrm{t}$ \\
\hline
\end{tabular}

${ }^{a}$ Bold type added to assist visual alignment of sequences. SNP = single nucleotide polymorphism. 
HAG4 is present in Setaria and Oryza pathogen populations and was also found in two Panicum repens isolate genome sequences (BTTrp-5 and BTTrp-6) (Soanes et al. 2017). WGS SNP analysis show that these Panicum repens isolates are outside of the Oryza and Setaria pathogen clade (Fig. 4) and more closely related to the Brachiaria Bd8401 isolate, sharing identical alleles for ten $H A G s$, of which two are unique to these isolates (Table 5). This suggests the two lineages (Fig. 4) may be derived from a common ancestor. Chromosome painting analysis of the Bd8401 sequence (Gladieux et al. 2018b) is consistent with genome-wide recombination involving the Setaria/Oryza lineage and apparently involving a partner with novel HAGs. HAG4 could have entered the Panicum repens lineage in a recombination event that generated the lineage containing Bd8401 and the Panicum repens isolates. However, the HAG4 allele found in BTTrp-5/BTTrp-6 isolates is the t $213 \mathrm{c}$ allele identical to a common allele in rice isolates. In the region from approximately $2,600 \mathrm{bp}$ upstream to approximately 760 bp downstream of the HAG4 start codon, most rice isolates have two to three SNPs relative to the Panicum repens isolates. This same region differed by a minimum of 10 SNPs from the seven Setaria isolate sequences. It is possible the HAG4 allele exists or existed in the Setaria pathogen population when recombination between Setaria and another lineage led to this
Panicum repens lineage; however, the close sequence identity between Panicum and Oryza isolate HAG4 sequences also suggests a possible recent movement of HAG4 from the rice isolate lineage into the Panicum repens lineage.

As mentioned before, the Br7 Triticum isolate has a different allele of HAG21 from all other members in the Lolium/Triticum group sequenced (Table 6). HAG21 is located approximately $124 \mathrm{~Kb}$ from the beginning of the chromosome 5 scaffold of the B71 Triticum isolate. Br7 DNA aligning to this region is most related to the Stenotaphrum sequence, based on chromosome painting analysis (Gladieux et al. 2018b). Using B71 as a scaffold, SSFL14-3 and BR7 aligned with high sequence identity from the beginning of the chromosome 5 scaffold until it began to diverge in the region from 2.5 to $10 \mathrm{~Kb}$ downstream of $H A G 21$. Thereafter, BR7 sequence aligned with high identity with B71 for at least $100 \mathrm{~Kb}$. Recombination that replaced a tip segment of chromosome 5 containing HAG21 appears to be the origin of this $H A G 21$ allele in $\mathrm{Br}$.

\section{DISCUSSION}

Expression and regulation of $H A G$ family members.

Updated annotation of the $H A G s$ allowed us to refine prior genome-wide transcriptome studies to assess $H A G$ expression patterns more comprehensively. Most genes were induced in

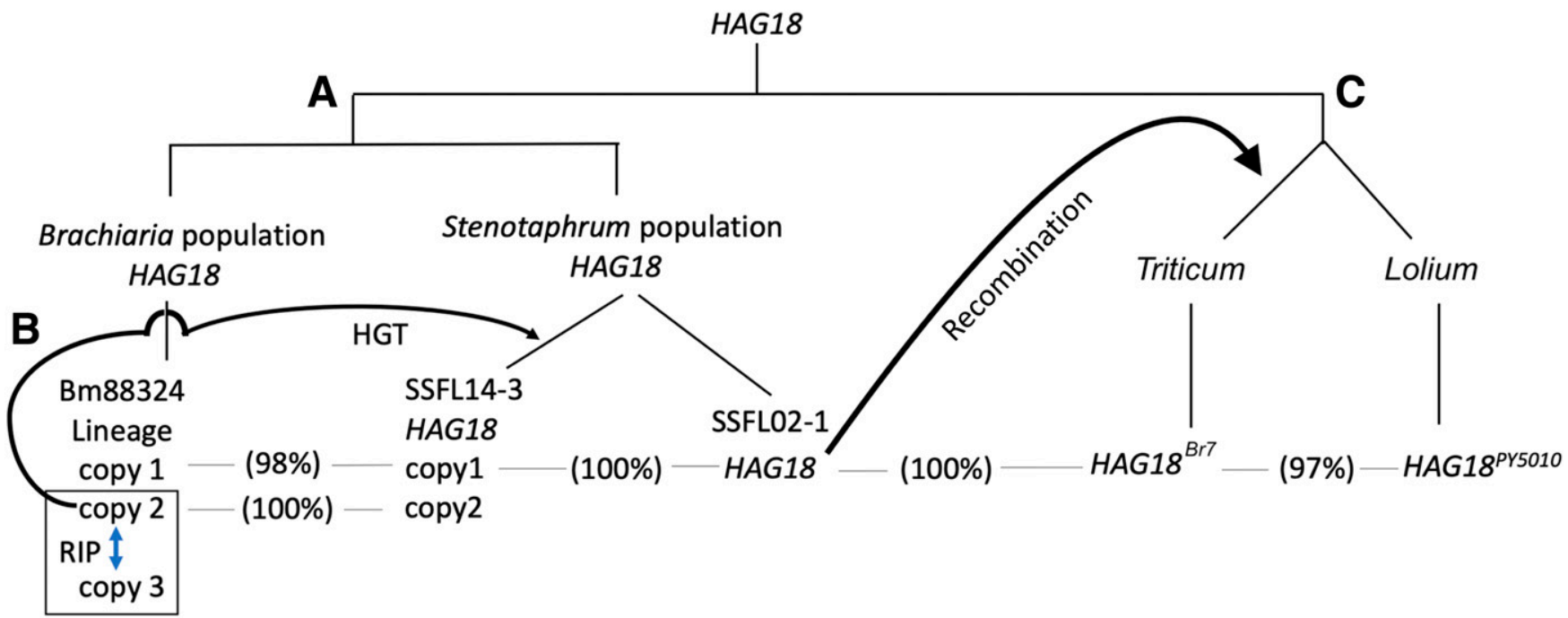

Fig. 5. Model for HAG18 evolution and movement. A, Populations diverge yielding Brachiaria and Stenotaphrum pathogen lineages with HAG18 orthologs (copy 1) with 98\% sequence identity. In the Bm88324-containing lineage, duplication generates three copies of the gene and copies 2 and 3 underwent repeatinduced point (RIP) mutagenesis. B, Copy 2 is transferred to a member of the Stenotaphrum lineage yielding the lineage containing SSFL14-3, resulting in the observed nearly $100 \%$ identity between copy 2 of Bm88324 and copy 2 of SSFL14-3. C, Recombination of the Stenotaphrum lineage with a progenitor of the Triticum lineage delivers the Stenotaphrum-like HAG18 copy 1 found in most Triticum lineage isolates, such as isolate Br7 (HAG18 $\left.{ }^{B r 7}\right)$ having nearly $100 \%$ identity with the Stenotaphrum isolate sequence. The Lolium population and some members of the Triticum population have an allele common to Lolium isolates with approximately $97 \%$ identity to the Br7 allele.

Table 7. Allele sequence and CG to TA transition counts for HAG4 indicative of repeat-induced point mutagenesis

\begin{tabular}{|c|c|c|c|c|}
\hline \multirow{3}{*}{$\begin{array}{l}\text { Isolate } \\
\text { US71 } \\
\text { MG08 (copy 1) }\end{array}$} & \multirow{3}{*}{$\begin{array}{l}\text { HAG4 allele (SNPs relative to P131) })^{\mathbf{a}} \\
\mathbf{t 2 1 3 c} \text { t267c } \\
\text { c22t c27t c32t g57a t66c g130a a147c t213c } \\
\text { c254t t267c }\end{array}$} & \multicolumn{3}{|c|}{ SNPs versus US71 ${ }^{b}$} \\
\hline & & CG to TA & \multirow{3}{*}{ MG08 } & \multirow{2}{*}{ Other } \\
\hline & & & & \\
\hline MG08 (copy 2) & $\begin{array}{l}\text { c22t c27t c32t c63t t66e g118a g130a g131a } \\
\text { t132g a147c t213c c231t t267c }\end{array}$ & 138 & & 5 \\
\hline MG08 (copy 3) & $\begin{array}{l}\text { g52a t66c g82a g109a a147c c210t t213c } \\
\text { t267c c270t g279a c297t }\end{array}$ & \multirow{4}{*}{130} & \multirow{4}{*}{ MG05 } & \multirow{4}{*}{5} \\
\hline MG05 (copy 1) & Identical to MG08 (copy 1) & & & \\
\hline MG05 (copy 2) & Identical to MG08 (copy 2) & & & \\
\hline MG05 (copy 3) & $\begin{array}{l}\text { g57a a147c c210t t213c t267e c270t g279a } \\
\text { c297t }\end{array}$ & & & \\
\hline
\end{tabular}


KJ201 (Jeon et al. 2020), with a time course similar to wellstudied effectors such as AvrPiz-t and AvrPi9 (Li et al. 2009; Wu et al. 2015). $P M K 1$ acts in planta to regulate critical functions required for host colonization (Sakulkoo et al. 2018). Combining analyses, HAG3, HAG12, HAG16, and the HAG5 pseudogenes are strongly dependent on $P M K 1$, and other family members (HAG8, HAG10, and HAG18) are probably regulated by $P M K 1$, but deeper sequencing and analyses of additional strains are needed. HAG4, HAG13, and HAG17 were not strongly influenced by $P M K 1$. Additional expression data combined with comparisons of promoter regions of the genes within and between populations would help to define candidate cis-acting elements responsible for the different patterns of regulation.

Our analysis uncovered the potential for additional transcriptional and posttranscriptional mechanisms controlling $H A G$ expression. Antisense RNA of the key regulator of the Neurospora circadian clock is regulated in antiphase to the sense transcript and leads to dicer-independent small interfering RNA production, stalling of RNA polymerase, and methylation of both histone $\mathrm{H} 3$ and DNA in the promoter of the gene (Dang et al. 2016; Xue et al. 2014). This process involves a conserved exonuclease (ER-1, enhanced RNA interference) that also functions in the cytoplasm to regulate stability of target RNAs. If a similar mechanism applies to the genes in which antisense transcripts were detected, HAG17 and, possibly, HAG1 and HAG13 ER-1 mutants would be predicted to have defects in their expression. Naturally occurring antisense RNAs are one form of long noncoding RNA. Long noncoding RNAs are found in the genomic regions associated with effector genes in Phytophthora spp. (Wang et al. 2018) and Ustilago maydis (Goulet et al. 2020). In $U$. maydis, antisense RNAs were implicated in regulating expression at unlinked loci, and disruption of one antisense RNA reduced aggressiveness of the pathogen.

The transcripts of several family members were mostly unspliced during growth in vitro. Since transcription of HAGs is very low in mycelia, it seems redundant or unnecessary to regulate intron splicing. However, if the low expression is a result of an antisense RNA regulatory mechanism, the unspliced RNA detected may be from the antisense RNA rather than $H A G$ transcript. Alternatively, intron retention has been shown to occur in plant RNAs and leads to retention in the nucleus prior to posttranscriptional splicing (Jia et al. 2020). This has been proposed to serve as a means of environmental or developmental regulation. Quantification of sense versus antisense transcripts in vitro and during colonization will help to address this question. Protein arginine methylation has been shown to impact splicing in $P$. oryzae and might therefore be involved in the regulated splicing of the HAGs or their antisense transcripts. Mutants of the protein arginine methyltransferase in $P$. oryzae have reduced aggressiveness ( $\mathrm{Li}$ et al. 2020). The HAGs provide candidate genes to further characterize the role of unspliced and antisense RNAs in transcriptional and posttranscriptional control of effector gene expression in fungal pathogens.

$H A G$ pseudogenes were found in several $P$. oryzae populations. The HAG2 and HAG5 pseudogenes were expressed and regulated similarly to other genes. Pseudogene RNA along with transcripts with alternatively spliced or unspliced introns generate premature termination codons. Transcripts with premature termination codons have been found to take part in a genetic compensation response involved in transcriptional regulation of other family members within gene families in zebrafish (Ma et al. 2019). Thus, the possibility exists for selection to maintain certain null alleles to modulate expression of gene family members.

In addition to identifying that gene family members display expression patterns expected for effector genes, our analysis has suggested several novel mechanisms that may impact effector expression and regulation.

\section{Gene family null alleles.}

We expect at least partial functional redundancy among gene family members, and we found the primary variation in the rice pathogen population was that of presence-absence polymorphism. Effector/Avr genes may operate at a host cultivar level (presence-absence polymorphism within a host-adapted population) or host species level (presence-absence polymorphism between different host-adapted populations). We observed both of these patterns for members of the $H A G$ family. As this pattern is often associated with the presence of resistance genes in the host, HAGs may be useful tools for screening grass and cereal germplasm for resistance genes, and identification of such genes may facilitate effective resistance gene deployment strategies for blast diseases.

\section{Allelic variation.}

Duplication and divergence is an important mechanism for innovation of new gene function. Because RIP yields multiple CG to TA transition mutations, candidate recent RIP events may be identified. RIP has been detected in other fungal plant pathogens, as illustrated in Leptosphaeria maculans (Fudal et al. 2009), in which RIP events led to stop codons in an Avr gene and was responsible for generation of virulent isolates in the field. They found $4 \%$ to $9 \%$ nucleotide divergence with nearly all SNPs being GC to TA transitions.

Two of the copies of HAG18 in Bm88324 carry the signature of RIP, a process that occurs during mating. That one of these is almost identical to one of the HAG18 copies in SSFL14-3 suggests that the copy moved into the Stenotaphrum isolate lineage after the RIP event occurred within the Bm88324 lineage. Whether the RIP allele provided a selective advantage to the Stenotaphrum lineage isolates bearing it is an interesting question that could be addressed by functional comparison of HAG18 alleles. If the RIP allele does provide a fitness advantage, it would define one mechanism for generation of new alleles that eventually might replace the older version of the gene or expand the gene family. The exclusive presence of HAG4 in the Setaria/Oryza lineage and apparent subsequent entry into the Panicum repens lineage suggests such an expansion is possible.

A second apparent example of RIP in three copies of HAG4 in two Setaria isolates suggests that RIP may be a common mechanism for diversification of $H A G$ family members. We observed that about $4 \%$ of the nucleotides were altered in the HAG4 region relative to US71 and $96.5 \%$ of these were CG to AT transitions, similar to the level of RIP mutation found for Leptosphaeria maculans. For RIP to contribute to sequence divergence, the occurrence of duplicated copies of effector genes may not be required. Many effector genes, including the HAGs, lie near repetitive sequences. RIP can spread beyond repetitive regions into single-copy sequences (Vyas and Kasbekar 2005). Multiple synonymous and nonsynonymous substitutions will arise simultaneously in RIP, complicating evaluation of gene evolution based on an assumption of one nucleotide change at a time.

\section{Recombination to generate $H A G$ haplotype diversity.}

In the rice population, evidence was found for sexual recombination at the center of origin, although the frequency of sexual recombination is low (Saleh et al. 2012; Zeigler 1998). Parasexual recombination in $P$. oryzae has been demonstrated in the laboratory (Noguchi et al. 2006) and strongly suggested to occur in the field (Zeigler 1998). Recombination between host-adapted populations also occurs (Gladieux et al. 2018a and b; Zeigler 1998).

Movement of mini-chromosomes can carry virulence determinants between strains (Peng et al. 2019). Movement of smaller regions associated with transposons has been shown for 
Avr-Pita, a gene that occurs in zero to three copies in P. oryzae (Chuma et al. 2011). The involvement of transposons in virulence factor transfers also has been shown in other fungal pathogen systems (McDonald et al. 2019). The Avr-Pita case suggests movement between sites within the genome and shuttling with mini-chromosomes. This mechanism is consistent with the cases where multicopy $H A G$ genes were observed, including HAG4, HAG12, HAG13, HAG18, and HAG21.

In Emericella nidulans, parasexual recombination frequency is much lower than meiotic recombination frequency (SouzaJúnior et al. 2007), and a low frequency of crossovers will result in larger segments of DNA being exchanged than during meiosis. The approximately 5-Mb block noted for the chromosome 1 region of isolate $87-120$ is consistent with parasexual recombination. Vegetative incompatibility could limit hyphal fusion of isolates and inhibit exchange of mini-chromosomes or the frequency of parasexual events; however, vegetative incompatibility appears to be weak in rice isolates and rice-Eleusine isolate pairings (Crawford et al. 1986; Zeigler 1998); thus, there may be no significant barrier to fusion in planta in mixed infections or during saprophytic phases (Avila-Adame 2014).

The $H A G$ family illustrates many of the mechanisms documented for movement of known fungal effector/Avr genes and provides specific examples that further amplifies studies showing that gene flow between $P$. oryzae populations occurs at easily measured frequency (Gladieux et al. 2018b). In addition to the examples illustrated here, examples of $H A G$ movement or recombination are evident in the $H A G$ haplotypes listed in Supplementary Table S3. Assuming that acquisition of an additional copy of a $H A G$ family member or a new $H A G$ allele provides, at most, a small advantage to the recipient, it is surprising that examples of RIP and $H A G$ movement between populations were so readily detected. It is possible that additional genes contributing to pathogen fitness may also be transferred with HAGs and this may facilitate the success of the recipient lineages. However, the observation of movement of $H A G$ alleles between populations suggest that $H A G s$ do play a role in parasitism, and the finding that combinations of $H A G$ alleles appear preferentially in some populations likely reflects that these combinations of alleles contribute to adaptation to that host. Competition between HAGs and their alleles could lead to replacement of less-fit alleles, and perhaps that is happening with the Stenotaphrum HAG18 allele observed in the Triticum population.

\section{MATERIALS AND METHODS}

\section{Identification of $H A G$ genes, SNPs, and repetitive sequences.}

We analyzed alignments of protein sequences to define gene family members and aligned DNA sequences to identify SNPs against the P131 rice isolate sequences for most genes, except we used SV9610 isolate sequences for HAG2,HAG5, and HAG6. SNPs identified in alignments of genomic DNA were mapped to the coding sequence (intron removed) to define alleles. SNPs were detected using an in-house PERL script to scan sequence alignments and to output the SNPs in the notation used here, e.g., a18c indicates the 'a' residue at position 18 of the coding sequence was changed to a 'c' residue. Small insertions and deletions were noted as position with the string of nucleotides inserted or deleted (indel). This is designated in Table 4, for example, as Del 212a to indicate a deletion at position 212 of a single 'a' residue. Indels were rare and alignments were examined in these cases to manually verify the calls and, if available, deposited sequencing reads for those genomes were queried to validate indels in the primary reads. Although rare mutations in introns that would prevent RNA splicing and thus represent null alleles were noted, we did not use this information in defining alleles for each gene. Repetitive sequences were defined as alignment of five or more copies of a sequence of at least $200 \mathrm{bp}$ in length.

\section{Analysis of RNA expression and intron splicing.}

Magic-BLAST (Boratyn et al. 2019) was used to align transcriptome sequence reads (Supplementary Table S6) obtained from the National Center for Biotechnology Information Short Read Archive database and these results were used to quantitate expression level and to assess introns. An inhouse PERL script was used to capture Magic-BLAST output and process the read information with respect to paired-end reads and intron/exon boundary overlaps. Fragments (pairedread fragments plus unpaired reads) were counted, and expression was assessed as fragments per kilobase of sequence (HAG coding region plus $150 \mathrm{bp}$ of flanking sequence) per million mapped reads (Jeon et al. 2020; Sakulkoo et al. 2018). Fragment sequences that crossed at least one intron boundary were counted as a splice or 'unsplice' event. Reads that either captured the spliced intron (called by MagicBLAST) or sequence alignments that either read through the intron boundary or were arrested at the intron/exon junction and had overhangs of unaligned sequence were captured. The captured information on these paired reads were compared and the read overlapping the intron was collected and counted as an intron overlapping read. When both reads overlapped the intron boundaries, only one read was counted as an intron overlapping read. Information about these reads combining Magic-BLAST output and intron/exon boundary information were then imported into a spreadsheet and were scanned manually to accept or reject intron overlapping reads. The main cause of rejection was due to sequences with arrested alignment at an intron/exon boundary that had overhangs that were ambiguous (e.g., single nucleotide overhang that could match to either spliced or unspliced intron) or the overhang beyond the intron/exon boundary failed to align with the predicted properly spliced sequence. Other than antisense intron reads, which were retained separately, all other reads with overhang sequence that did not align to the properly spliced intron were rejected. These rare, rejected reads were due to alternative splicing or, for some overhang sequences, an alignment to nearby sequence was not found. The error rate for manual retention and rejection of intron overlapping reads was not assessed.

There was no false positive signal for $H A G$ genes from alignments to uninfected rice tissue in the KJ201 time course data. We empirically determined that rejecting Magic-BLAST alignments matching less than $50 \%$ of the read length and alignments that displayed less than $92 \%$ identity across the alignment prevented cross-alignment of reads to different $H A G$ family members.

To assess intron splicing efficiency and antisense transcripts, raw counts of spliced and unspliced reads were used. Compared with controls, $H A G$ family expression was not significantly different in response to PKC inhibition or in the vrfl mutant versus wild type. The control and test transcriptome data for mycelia in the PKC study and for appressoria of both 70-15 and vrfl were combined to assess spliced versus unspliced RNA (Table 3).

\section{Characterization of genome sequences and assemblies.}

Errors in genome assemblies are difficult to avoid due to repetitive elements and duplicated regions. Rare alleles were checked where possible, e.g., sequencing read archive data (Supplementary Table S6) were used as needed to assess sequence quality of SNPs, indels, and the B71 assembly by manual inspection and assembly. 
In cases in which the coding region was only partially covered by a single contig, or coverage involved multiple contigs where an individual contig did not completely contain the coding region, we assessed whether this was due to insertion or deletion and these cases are noted in Supplementary Table S3. If no determination could be made with the assembly, these alleles were indicated in Supplementary Table S3 as 'incomplete.' Sixteen of the 20 incomplete genes were found in four of the genome assemblies. HAG4 was absent from the B157 genome assembly; however, we probed the fastq sequence files and identified a single read that had a perfect match for HAG4. Therefore, we cannot be certain about the presence or absence of HAG4 in this isolate. Twelve HAGs were absent from the RMgDI genome assembly. However, transcriptome data submitted along with the genome assembly for the isolate was assessed and we identified RNA for eight of the missing 12 genes in those data. This is noted in Supplementary Table S3. Chromatogram and sequence read IDs used to assess the HAG3 sequence of 7015 are listed in Supplementary Table S7.

Visualization of HAG genes on chromosomes was done using PhenoGram (Wolfe et al. 2013). The consensus graphic was prepared using WebLogo (Schneider and Stephens 1990).

\section{ACKNOWLEDGMENTS}

We thank H. Wilkinson for helpful comments on the manuscript.

\section{LITERATURE CITED}

Almagro Armenteros, J. J., Tsirigos, K. D., Sønderby, C. K., Petersen, T. N., Winther, O., Brunak, S., von Heijne, G., and Nielsen, H. 2019. SignalP 5.0 improves signal peptide predictions using deep neural networks. Nat. Biotechnol. 37:420-423.

Altschul, S. F., Gish, W., Miller, W., Myers, E. W., and Lipman, D. J. 1990. Basic local alignment search tool. J. Mol. Biol. 215:403-410.

Asuke, S., Nishimi, S., and Tosa, Y. 2020a. At least five major genes are involved in the avirulence of an Eleusine isolate of Pyricularia oryzae on common wheat. Phytopathology 110:465-471.

Asuke, S., Tanaka, M., Hyon, G. S., Inoue, Y., Vy, T. T. P., Niwamoto, D., Nakayashiki, H., and Tosa, Y. 2020b. Evolution of an Eleusine-specific subgroup of Pyricularia oryzae through a gain of an avirulence gene. Mol. Plant-Microbe Interact. 33:153-165.

Avila-Adame, C. 2014. Transmission of the G143A QoI-resistance point mutation through anastomosis in Magnaporthe grisea. Pest Manag. Sci. 70:1918-823

Bao, J., Chen, M., Zhong, Z., Tang, W., Lin, L., Zhang, X., Jiang, H., Zhang, D., Miao, C., Tang, H., Zhang, J., Lu, G., Ming, R., Norvienyeku, J., Wang, B., and Wang, Z. 2017. PacBio sequencing reveals transposable elements as a key contributor to genomic plasticity and virulence variation in Magnaporthe oryzae. Mol. Plant 10:1465-1468

Boratyn, G. M., Thierry-Mieg, J., Thierry-Mieg, D., Busby, B., and Madden, T. L. 2019. Magic-BLAST, an accurate RNA-seq aligner for long and short reads. BMC Bioinformatics 20:405.

Brown, N. P., Leroy, C., and Sander, C. 1998. MView: A web-compatible database search or multiple alignment viewer. Bioinformatics 14: 380-381.

Cao, H., Huang, P., Zhang, L., Shi, Y., Sun, D., Yan, Y., Liu, X., Dong, B., Chen, G., Snyder, J. H., Lin, F., and Lu, J. 2016. Characterization of 47 Cys2 -His2 zinc finger proteins required for the development and pathogenicity of the rice blast fungus Magnaporthe oryzae. New Phytol. 211:1035-1051

Chiapello, H., Mallet, L., Guérin, C., Aguileta, G., Amselem, J., Kroj, T., Ortega-Abboud, E., Lebrun, M. H., Henrissat, B., Gendrault, A., Rodolphe, F., Tharreau, D., and Fournier, E. 2015. Deciphering genome content and evolutionary relationships of isolates from the fungus Magnaporthe oryzae attacking different host plants. Genome Biol. Evol. 7:2896-2912.

Chuma, I., Isobe, C., Hotta, Y., Ibaragi, K., Futamata, N., Kusaba, M., Yoshida, K., Terauchi, R., Fujita, Y., Nakayashiki, H., Valent, B., and Tosa, Y. 2011. Multiple translocation of the AVR-Pita effector gene among chromosomes of the rice blast fungus Magnaporthe oryzae and related species. PLoS Pathog. 7:e1002147.
Couch, B. C., and Kohn, L. M. 2002. A multilocus gene genealogy concordant with host preference indicates segregation of a new species, Magnaporthe oryzae, from M. grisea. Mycologia 94:683-693.

Crawford, M. S., Chumley, F. G., Weaver, C. G., and Valent, B. 1986. Characterization of the heterokaryotic and vegetative diploid phases of Magnaporthe grisea. Genetics 114:1111-1129.

Dang, Y., Cheng, J., Sun, X., Zhou, Z., and Liu, Y. 2016. Antisense transcription licenses nascent transcripts to mediate transcriptional gene silencing. Genes Dev. 30:2417-2432.

Dean, R. A., Talbot, N. J., Ebbole, D. J., Farman, M. L., Mitchell, T. K., Orbach, M. J., Thon, M., Kulkarni, R., Xu, J. R., Pan, H., Read, N. D., Lee, Y. H., Carbone, I., Brown, D., Oh, Y. Y., Donofrio, N., Jeong, J. S. Soanes, D. M., Djonovic, S., Kolomiets, E., Rehmeyer, C., Li, W. Harding, M., Kim, S., Lebrun, M. H., Bohnert, H., Coughlan, S., Butler J., Calvo, S., Ma, L. J., Nicol, R., Purcell, S., Nusbaum, C., Galagan, J. E., and Birren, B. W. 2005. The genome sequence of the rice blast fungus Magnaporthe grisea. Nature 434:980-986.

Dong, S., Stam, R., Cano, L. M., Song, J., Sklenar, J., Yoshida, K., Bozkurt, T. O., Oliva, R., Liu, Z., Tian, M., Win, J., Banfield, M. J., Jones, A. M., van der Hoorn, R. A., and Kamoun, S. 2014. Effector specialization in a lineage of the Irish potato famine pathogen. Science 343:552-555.

Fudal, I., Ross, S., Brun, H., Besnard, A. L., Ermel, M., Kuhn, M. L., Balesdent, M. H., and Rouxel, T. 2009. Repeat-induced point mutation (RIP) as an alternative mechanism of evolution toward virulence in Leptosphaeria maculans. Mol. Plant-Microbe Interact. 22:932-941.

Gladieux, P., Condon, B., Ravel, S., Soanes, D., Maciel, J. L. N., Nhani, A., Jr., Chen, L., Terauchi, R., Lebrun, M. H., Tharreau, D., Mitchell, T. Pedley, K. F., Valent, B., Talbot, N. J., Farman, M., and Fournier, E. 2018b. Gene flow between divergent cereal- and grass-specific lineages of the rice blast fungus Magnaporthe oryzae. MBio 9:e01219-17.

Gladieux, P., Ravel, S., Rieux, A., Cros-Arteil, S., Adreit, H., Milazzo, J., Thierry, M., Fournier, E., Terauchi, R., and Tharreau, D. 2018a. Coexistence of multiple endemic and pandemic lineages of the rice blast pathogen. MBio 9:e01806-17.

Gómez Luciano, L. B., Tsai, I. J., Chuma, I., Tosa, Y., Chen, Y. H., Li, J. Y., Li, M. Y., Lu, M. J., Nakayashiki, H., and Li, W. H. 2019. Blast fungal genomes show frequent chromosomal changes, gene gains and losses, and effector gene turnover. Mol. Biol. Evol. 36:1148-1161.

Goulet, K. M., Storfie, E. R. M., and Saville, B. J. 2020. Exploring links between antisense RNAs and pathogenesis in Ustilago maydis through transcript and gene characterization. Fungal Genet. Biol. 134:103283.

Ikeda, K., Nakayashiki, H., Kataoka, T., Tamba, H., Hashimoto, Y., Tosa, Y., and Mayama, S. 2002. Repeat-induced point mutation (RIP) in Magnaporthe grisea: Implications for its sexual cycle in the natural field context. Mol. Microbiol. 45:1355-1364.

Inoue, Y., Vy, T. T. P., Yoshida, K., Asano, H., Mitsuoka, C., Asuke, S., Anh, V. L., Cumagun, C. J. R., Chuma, I., Terauchi, R., Kato, K., Mitchell, T., Valent, B., Farman, M., and Tosa, Y. 2017. Evolution of the wheat blast fungus through functional losses in a host specificity determinant. Science 357:80-83.

Jeon, J., Lee, G. W., Kim, K. T., Park, S. Y., Kim, S., Kwon, S., Huh, A., Chung, H., Lee, D. Y., Kim, C. Y., and Lee, Y. H. 2020. Transcriptome profiling of the rice blast fungus Magnaporthe oryzae and its host Oryza sativa during infection. Mol. Plant-Microbe Interact. 33:141-144.

Jia, J., Long, Y., Zhang, H., Li, Z., Liu, Z., Zhao, Y., Lu, D., Jin, X., Deng, X., Xia, R., Cao, X., and Zhai, J. 2020. Post-transcriptional splicing of nascent RNA contributes to widespread intron retention in plants. Nat. Plants 6:780-788.

Kang, S., Sweigard, J. A., and Valent, B. 1995. The PWL host specificity gene family in the blast fungus Magnaporthe grisea. Mol. Plant-Microbe Interact. 8:939-948.

Kim, K. T., Ko, J., Song, H., Choi, G., Kim, H., Jeon, J., Cheong, K., Kang, S., and Lee, Y. H. 2019. Evolution of the genes encoding effector candidates within multiple pathotypes of Magnaporthe oryzae. Front. Microbiol. 10:2575.

Kimura, M., and Yamaguchi, I. 1998. Convergent transcription units and their promoters at both ends of pot2, an inverted repeat transposon from the rice blast fungus. J. Biochem. 124:268-273.

Kito, H., Takahashi, Y., Sato, J., Fukiya, S., Sone, T., and Tomita, F. 2003. Occan, a novel transposon in the Fot1 family, is ubiquitously found in several Magnaporthe grisea isolates. Curr. Genet. 42:322-331.

Latorre, S. M., Reyes-Avila, C. S., Malmgren, A., Win, J., Kamoun, S., and Burbano, H. A. 2020. Differential loss of effector genes in three recently expanded pandemic clonal lineages of the rice blast fungus. BMC Biol 18:88.

Lawson, D. J., Hellenthal, G., Myers, S., and Falush, D. 2012. Inference of population structure using dense haplotype data. PLoS Genet. 8: e1002453. 
Li, W., Wang, B., Wu, J., Lu, G., Hu, Y., Zhang, X., Zhang, Z., Zhao, Q., Feng, Q., Zhang, H., Wang, Z., Wang, G., Han, B., Wang, Z., and Zhou, B. 2009. The Magnaporthe oryzae avirulence gene AvrPiz-t encodes a predicted secreted protein that triggers the immunity in rice mediated by the blast resistance gene Piz-t. Mol. Plant-Microbe Interact. 22: 411-420.

Li, Z., Wu, L., Wu, H., Zhang, X., Mei, J., Zhou, X., Wang, G. L., and Liu, W. 2020. Arginine methylation is required for remodelling pre-mRNA splicing and induction of autophagy in rice blast fungus. New Phytol. 225:413-429.

Liao, J., Huang, H., Meusnier, I., Adreit, H., Ducasse, A., Bonnot, F., Pan, L., He, X., Kroj, T., Fournier, E., Tharreau, D., Gladieux, P., and Morel, J. B. 2016. Pathogen effectors and plant immunity determine specialization of the blast fungus to rice subspecies. eLife 5:e19377.

Ma, Z., Zhu, P., Shi, H., Guo, L., Zhang, Q., Chen, Y., Chen, S., Zhang, Z., Peng, J., and Chen, J. 2019. PTC-bearing mRNA elicits a genetic compensation response via Upf3a and COMPASS components. Nature 568:259-263.

Madeira, F., Park, Y. M., Lee, J., Buso, N., Gur, T., Madhusoodanan, N., Basutkar, P., Tivey, A. R. N., Potter, S. C., Finn, R. D., and Lopez, R. 2019. The EMBL-EBI search and sequence analysis tools APIs in 2019. Nucleic Acids Res. 47 (W1):W636-W641.

McDonald, M. C., Taranto, A. P., Hill, E., Schwessinger, B., Liu, Z., Simpfendorfer, S., Milgate, A., and Solomon, P. S. 2019. Transposonmediated horizontal transfer of the host-specific virulence protein ToxA between three fungal wheat pathogens. MBio 10:e01515-19.

Noguchi, M. T., Yasuda, N., and Fujita, Y. 2006. Evidence of genetic exchange by parasexual recombination and genetic analysis of pathogenicity and mating type of parasexual recombinants in rice blast fungus, Magnaporthe oryzae. Phytopathology 96:746-750.

Park, C. H., Chen, S., Shirsekar, G., Zhou, B., Khang, C. H., Songkumarn, P., Afzal, A. J., Ning, Y., Wang, R., Bellizzi, M., Valent, B., and Wang, G. L. 2012. The Magnaporthe oryzae effector AvrPiz-t targets the RING E3 ubiquitin ligase APIP6 to suppress pathogen-associated molecular pattern-triggered immunity in rice. Plant Cell 24: 4748-4762.

Peng, Z., Oliveira-Garcia, E., Lin, G., Hu, Y., Dalby, M., Migeon, P., Tang, H., Farman, M., Cook, D., White, F. F., Valent, B., and Liu, S. 2019. Effector gene reshuffling involves dispensable mini-chromosomes in the wheat blast fungus. PLoS Genet. 15:e1008272.

Penn, T. J., Wood, M. E., Soanes, D. M., Csukai, M., Corran, A. J., and Talbot, N. J. 2015. Protein kinase C is essential for viability of the rice blast fungus Magnaporthe oryzae. Mol. Microbiol. 98:403-419.

Petit-Houdenot, Y., Langner, T., Harant, A., Win, J., and Kamoun, S. 2020. A clone resource of Magnaporthe oryzae effectors that share sequence and structural similarities across host-specific lineages. Mol. PlantMicrobe Interact. 33:1032-1035.

Pieck, M. L., Ruck, A., Farman, M. L., Peterson, G. L., Stack, J. P., Valent, B., and Pedley, K. F. 2017. Genomics-based marker discovery and diagnostic assay development for wheat blast. Plant Dis. 101:103-109.

Sakulkoo, W., Osés-Ruiz, M., Oliveira Garcia, E., Soanes, D. M., Littlejohn, G. R., Hacker, C., Correia, A., Valent, B., and Talbot, N. J. 2018. A single fungal MAP kinase controls plant cell-to-cell invasion by the rice blast fungus. Science 359:1399-1403.

Saleh, D., Xu, P., Shen, Y., Li, C., Adreit, H., Milazzo, J., Ravigné, V., Bazin, E., Nottéghem, J. L., Fournier, E., and Tharreau, D. 2012. Sex at the origin: An Asian population of the rice blast fungus Magnaporthe oryzae reproduces sexually. Mol. Ecol. 21:1330-1344.

Schneider, T. D., and Stephens, R. M. 1990. Sequence logos: A new way to display consensus sequences. Nucleic Acids Res. 18:6097-6100.
Soanes, D., Ryder, L., Islam, M., and Talbot, N. J. 2017. Genome assemblies of Magnaporthe oryzae isolated from Bangladesh in 2016 and 2017. figshare. Published online.

Souza-Júnior, S. A., Becker, T. C., and Castro-Prado, M. A. 2007. Asexual recombination in a $u v s H$ mutant of Aspergillus nidulans. Biol. Res. 40:65-71.

Sweigard, J. A., Carroll, A. M., Kang, S., Farrall, L., Chumley, F. G., and Valent, B. 1995. Identification, cloning, and characterization of $P W L 2$, a gene for host species specificity in the rice blast fungus. Plant Cell 7:1221-1233.

Torres, M. F., Ghaffari, N., Buiate, E. A., Moore, N., Schwartz, S., Johnson, C. D., and Vaillancourt, L. J. 2016. A Colletotrichum graminicola mutant deficient in the establishment of biotrophy reveals early transcriptional events in the maize anthracnose disease interaction. BMC Genomics 17:202.

Tosa, Y., Osue, J., Eto, Y., Oh, H. S., Nakayashiki, H., Mayama, S., and Leong, S. A. 2005. Evolution of an avirulence gene, AVR1-CO39, concomitant with the evolution and differentiation of Magnaporthe oryzae. Mol. Plant-Microbe Interact. 18:1148-1160.

Vyas, M., and Kasbekar, D. P. 2005. Collateral damage: Spread of repeatinduced point mutation from a duplicated DNA sequence into an adjoining single-copy gene in Neurospora crassa. J. Biosci. 30:15-20.

Wang, Y., Ye, W., and Wang, Y. 2018. Genome-wide identification of long non-coding RNAs suggests a potential association with effector gene transcription in Phytophthora sojae. Mol. Plant Pathol. 19:2177-2186.

Weßling, R., Epple, P., Altmann, S., He, Y., Yang, L., Henz, S. R., McDonald, N., Wiley, K., Bader, K. C., Gläßer, C., Mukhtar, M. S., Haigis, S., Ghamsari, L., Stephens, A. E., Ecker, J. R., Vidal, M., Jones, J. D., Mayer, K. F., Ver Loren van Themaat, E., Weigel, D., SchulzeLefert, P., Dangl, J. L., Panstruga, R., and Braun, P. 2014. Convergent targeting of a common host protein-network by pathogen effectors from three kingdoms of life. Cell Host Microbe 16:364-375.

Wolfe, D., Dudek, S., Ritchie, M. D., and Pendergrass, S. A. 2013. Visualizing genomic information across chromosomes with PhenoGram. BioData Min. 6:18.

Wu, J., Kou, Y., Bao, J., Li, Y., Tang, M., Zhu, X., Ponaya, A., Xiao, G., Li, J., Li, C., Song, M. Y., Cumagun, C. J., Deng, Q., Lu, G., Jeon, J. S., Naqvi, N. I., and Zhou, B. 2015. Comparative genomics identifies the Magnaporthe oryzae avirulence effector AvrPi9 that triggers Pi9mediated blast resistance in rice. New Phytol. 206:1463-1475.

Xue, M., Yang, J., Li, Z., Hu, S., Yao, N., Dean, R. A., Zhao, W., Shen, M., Zhang, H., Li, C., Liu, L., Cao, L., Xu, X., Xing, Y., Hsiang, T., Zhang, Z., $\mathrm{Xu}$, J. R., and Peng, Y. L. 2012. Comparative analysis of the genomes of two field isolates of the rice blast fungus Magnaporthe oryzae. PLoS Genet. 8:e1002869.

Xue, Z., Ye, Q., Anson, S. R., Yang, J., Xiao, G., Kowbel, D., Glass, N. L., Crosthwaite, S. K., and Liu, Y. 2014. Transcriptional interference by antisense RNA is required for circadian clock function. Nature 514:650-653.

Yoshida, K., Saunders, D. G., Mitsuoka, C., Natsume, S., Kosugi, S., Saitoh, H., Inoue, Y., Chuma, I., Tosa, Y., Cano, L. M., Kamoun, S., and Terauchi, R. 2016. Host specialization of the blast fungus Magnaporthe oryzae is associated with dynamic gain and loss of genes linked to transposable elements. BMC Genomics 17:370.

Zeigler, R. S. 1998. Recombination in Magnaporthe grisea. Annu. Rev. Phytopathol. 36:249-275.

Zhong, Z., Chen, M., Lin, L., Chen, R., Liu, D., Norvienyeku, J., Zheng, H., and Wang, Z. 2020. Genetic variation bias toward noncoding regions and secreted proteins in the rice blast fungus Magnaporthe oryzae. mSystems 5:e00346-20.

Zhong, Z., Chen, M., Lin, L., Han, Y., Bao, J., Tang, W., Lin, L., Lin, Y., Somai, R., Lu, L., Zhang, W., Chen, J., Hong, Y., Chen, X., Wang, B., Shen, W. C., Lu, G., Norvienyeku, J., Ebbole, D. J., and Wang, Z. 2018. Population genomic analysis of the rice blast fungus reveals specific events associated with expansion of three main clades. ISME J. 12:1867-1878. 